\title{
An Expression of Harmonic Vector Fields on Hyperbolic 3-Cone-Manifolds in Terms of Hypergeometric Functions
}

\author{
By \\ Michihiko FuJII* and Hiroyuki OCHIAI**
}

\begin{abstract}
Let $V$ be a neighborhood of a singular locus of a hyperbolic 3-cone-manifold, which is a quotient space of the 3-dimensional hyperbolic space. In this paper we give an explicit expression of a harmonic vector field $v$ on the hyperbolic manifold $V$ in terms of hypergeometric functions. The expression is obtained by solving a system of ordinary differential equations which is induced by separation of the variables in the vector-valued partial differential equation $(\Delta+4) \tau=0$, where $\Delta$ is the Laplacian of $V$ and $\tau$ is the dual 1-form of $v$. We transform this system of ordinary differential equations to single-component differential equations by elimination of unknown functions and solve these equations. The most important step in solving them consists of two parts, decomposing their differential operators into differential operators of the type appearing in Riemann's $P$-equation in the ring of differential operators and then describing the projections to the components of this decomposition in terms of differential operators that are also of the type appearing in Riemann's $P$-equation.
\end{abstract}

\section{$\S 1 . \quad$ Introduction}

Let $U$ be a 3 -dimensional space $\left\{(r, \theta, \phi) \in \mathbf{R}^{3} ; r>0\right\}$ with Riemannian metric $g:=d r^{2}+\sinh ^{2} r d \theta^{2}+\cosh ^{2} r d \phi^{2}$. The Riemannian space $U$ is a model

Communicated by T. Kobayashi. Received July 29, 2004. Revised November 14, 2006. 2000 Mathematics Subject Classification(s): Primary 16S32, 33C05, 34A30, 34Lxx; Secondary 57M50, 58J05.

Key Words: harmonic vector field, hyperbolic manifold, cone manifold, hypergeometric function, ordinary differential equation, Fuchsian type, Riemann's P-equation, ring of differential operators.

*Department of Mathematics, Kyoto University, Sakyo-ku, Kyoto 606-8502, Japan. e-mail: mfujii@math.kyoto-u.ac.jp

** Department of Mathematics, Nagoya University, Chikusa, Nagoya 464-0806, Japan. e-mail: ochiai@math.nagoya-u.ac.jp 
space of the 3-dimensional hyperbolic space. Now, let $\alpha, t$ and $l$ be real numbers satisfying that $\alpha>0$ and $l>0$. Let $V$ be a quotient space of $U$ defined by the equivalence relation (2) below. Then the space $V$ is homeomorphic to a product of an open interval and a 2-dimensional torus and the induced Riemannian metric on $V$ is the same form as $g$. This space $V$ is a model space of a neighborhood of a singular locus of a hyperbolic 3-cone-manifold whose cone angle is $\alpha$ and complex length along the singular locus is $l+t \sqrt{-1}$. Let $\Delta$ be the Laplacian of $V$ with respect to the induced Riemannian metric. We consider the vector-valued partial differential equation $\Delta v=0$, where $v$ is a vector field on $V$. Its solutions are called harmonic vector fields. Harmonic vector fileds play an important role to investigate infinitesimal deformations of hyperbolic cone-manifold structures.

In this paper we give an explicit expression of harmonic vector fields. We find out that harmonic vector fields can be expressed by hypergeometric functions. By taking the dual of $\Delta v=0$, we obtain the vector-valued partial differential equation $(\Delta+4) \tau=0$, where $\tau$ is the dual 1 -form of $v$. In this paper, we also consider a more general differential equation $(\Delta+2-\lambda) \tau=0$ which contains a parameter $\lambda \in \mathbf{R}$. In the case of harmonic vector fields, the parameter $\lambda$ is fixed to be a particular value, i.e., $\lambda=-2$.

By applying the method of separation of variables to $(\Delta+2-\lambda) \tau=0$, we obtain a system of ordinary differential equations in the variable $r$, given in (3) below. Next, we replace the independent variable $r$ by $z$, defined by $z=\left(\frac{\sinh r}{\cosh r}\right)^{2}$. Then we obtain a system of ordinary differential equations in $z$, given in (4) or (5) below. Most of the efforts of the paper is devoted to examine this system.

The system (5) of ordinary differential equations has two parameters, denoted by $a$ and $b$, which come from the given geometric parameters $\alpha, t$ and $l$. For the most general case, i.e., $a b \neq 0$, we can transform this to a singlecomponent ordinary differential equation by elimination of unknown functions. This is a 6 -th order homogeneous linear differential equation of Fuchsian type with three singular points, $z=0, z=1$ and $z=\infty$. For the degenerate case, i.e., $a b=0$, the system (5) are reduced, so treated separately. However we give the relations between the general case and the degenerated cases explicitly (see the beginning of Section 3). We show that the differential operators appearing in these single-component differential equations can be factorized into operators of the type appearing in Riemann's $P$-equation (see Theorems 3.1.1, 3.1.5, 3.2.1 and 3.3.1). The factorizations indicate the sub/quotient structure of the system. We expect more; the system may be decomposed into the direct 
sum of these factors. In our case, we can obtain such decompositions. We can construct (see Ref. [2] for the algorithmic point of view) the differential operators with a special property, each of which gives the projection operator onto the quotient factor (see Theorems 3.1.2 and 3.1.6). Due to these two results, we obtain expressions of solutions of the single-component ordinary differential equations under consideration in terms of those of Riemann's $P$-equations (see Corollaries 3.1.3, 3.1.7, 3.2.2 and 3.3.2).

We also represent fundamental systems of solutions to the system of ordinary differential equations in (5) in terms of those of Riemann's $P$-equations (see Propositions 4.1.3, 4.1.6, 4.2.1 and 4.3.1). Moreover, if we assume that the parameter $a$ satisfies a "generic" condition (see Assumptions 5.1.1 and 5.3.1), then we can express the fundamental systems of solutions to the system of differential equations in (5) explicitly (see Sections 5.1 and 5.3). The functions composing these fundamental systems are explicitly represented in terms of hypergeometric functions. Hence, substituting $z=\left(\frac{\sinh r}{\cosh r}\right)^{2}$ and $\lambda=-2$ into these fundamental systems, under the assumption expressed in Assumptions 5.1.1 and 5.3.1, we can express in terms of hypergeometric functions any 1form $\tau$ on $V$ that is obtained by separating the independent variables in the partial differential equation $(\Delta+4) \tau=0$. Then taking the dual of $\tau$, we obtain the desired expression for $v$, which is harmonic on $V$ (see Theorem 2.1).

In this paper, we have omitted complicated explicit forms of differential operators and long but straightforward computations for several formulae satisfied by these differential operators. One can obtain the detailed version [3] of this paper, where the explicit forms of such operators are described. Referring to [3], one can also verify by oneself almost all the computation in this paper by making use of the software Mathematica released from Wolfram Research. We give a text file on the website

$$
\text { http://www.math.kyoto-u.ac.jp/ mfujii/harmonic/operators.txt }
$$

where one can obtain all the differential operators written in Mathematica. After taking operators from the website above, by carrying out programs at the websites described in [3], one can check calculations.

The authors would like to thank Dr. Masaaki Suzuki for suggestions on programming. 


\section{§2. A System of Ordinary Differential Equations Providing Harmonic Vector Fields on Hyperbolic 3-Cone-Manifolds}

In this section we give background discussion on necessary concepts of differential geometry, obtain a system of ordinary differential equations whose solutions provide harmonic vector fields on a hyperbolic 3-cone-manifold and state our main theorem. (See Rosenberg [7] for a general reference on Riemannian geometry and Hodgson and Kerckhoff [4] for discussion specific to hyperbolic 3-cone-manifolds.)

Let $U$ be a 3 -dimensional space

$$
\left\{(r, \theta, \phi) \in \mathbf{R}^{3} ; r>0\right\}
$$

with Riemannian metric

$$
g:=d r^{2}+\sinh ^{2} r d \theta^{2}+\cosh ^{2} r d \phi^{2} .
$$

We define $\left(\omega_{1}, \omega_{2}, \omega_{3}\right):=(d r, \sinh r d \theta, \cosh r d \phi)$ as a co-frame on $U$. We denote by $\left(e_{1}, e_{2}, e_{3}\right)$ the orthonormal frame on $U$ dual to $\left(\omega_{1}, \omega_{2}, \omega_{3}\right)$. Then we have $e_{1}=\frac{\partial}{\partial r}, e_{2}=\frac{1}{\sinh r} \frac{\partial}{\partial \theta}$ and $e_{3}=\frac{1}{\cosh r} \frac{\partial}{\partial \phi}$. In this section, for notational convenience, we set $x^{1}=r, x^{2}=\theta$, and $x^{3}=\phi$. We express the metric $g$ as $\sum_{i, j=1}^{3} g_{i, j} d x^{i} \otimes d x^{j}$. Then we have $g_{1,1}=1, g_{2,2}=\sinh ^{2} x^{1}, g_{3,3}=\cosh ^{2} x^{1}$ and $g_{i, j}=0$ (if $i \neq j$ ). The Christoffel symbol $\Gamma_{j, k}^{i}$ can be calculated by using the formula

$$
\Gamma_{j, k}^{i}=\frac{1}{2} \sum_{l} g^{i, l}\left(\frac{\partial g_{j, l}}{\partial x^{k}}+\frac{\partial g_{k, l}}{\partial x^{j}}-\frac{\partial g_{j, k}}{\partial x^{l}}\right),
$$

where $\left(g^{k, l}\right)=\left(g_{i, j}\right)^{-1}$. The Levi-Civita connection $\nabla$ can be calculated from

$$
\nabla_{\frac{\partial}{\partial x^{j}}} \frac{\partial}{\partial x^{k}}=\sum_{i} \Gamma_{j, k}^{i} \frac{\partial}{\partial x^{i}}
$$

Direct calculation yields

$$
\left(\nabla_{\frac{\partial}{\partial x^{j}}} \frac{\partial}{\partial x^{k}}\right)=\left(\begin{array}{ccc}
0 & \frac{\cosh r}{\sinh r} \frac{\partial}{\partial \theta} & \frac{\sinh r}{\cosh r} \frac{\partial}{\partial \phi} \\
\frac{\cosh r}{\sinh r} \frac{\partial}{\partial \theta} & -\sinh r \cosh r \frac{\partial}{\partial r} & 0 \\
\frac{\sinh r}{\cosh r} \frac{\partial}{\partial \phi} & 0 & -\sinh r \cosh r \frac{\partial}{\partial r}
\end{array}\right)
$$

Also, for $\left(\omega_{\lambda}^{\mu}\right)$, the matrix of the connection 1-forms, we find

$$
\left(\omega_{\lambda}^{\mu}\right)=\left(\begin{array}{ccc}
0 & -\frac{\cosh r}{\sinh r} \omega_{2} & -\frac{\sinh r}{\cosh r} \omega_{3} \\
\frac{\cosh r}{\sinh r} \omega_{2} & 0 & 0 \\
\frac{\sinh r}{\cosh r} \omega_{3} & 0 & 0
\end{array}\right) .
$$


It can also be checked that $U$ is a space of constant sectional curvature -1 .

Let $\Omega^{p}(U)$ denote the space of smooth, real-valued $p$-forms on $U$. Let $d$ be the usual exterior derivative of smooth real-valued forms on $U$ :

$$
d: \Omega^{p}(U) \rightarrow \Omega^{p+1}(U) .
$$

We denote by $*$ the Hodge star operator defined by the Riemannian metric $g$ on $U$. Then we have

$$
g(\phi, * \psi) d U=\phi \wedge \psi,
$$

for any real-valued $p$-form $\phi$ and $(3-p)$-form $\psi$, where $d U$ is the volume form of $U$. Let $\delta$ be the adjoint of $d$ :

$$
\delta: \Omega^{p}(U) \rightarrow \Omega^{p-1}(U) .
$$

Denoting by $\Delta$ the Laplacian operating on smooth real-valued forms for the Riemannian manifold $U$, we have

$$
\Delta=d \delta+\delta d
$$

If we express a 1-form $\tau$ on $U$ as

$$
\tau=f(r, \theta, \phi) \omega_{1}+g(r, \theta, \phi) \omega_{2}+h(r, \theta, \phi) \omega_{3},
$$

then, by explicit computation, we obtain (see Ref. [4] pp. 26-27)

(1)

$$
\begin{aligned}
(\Delta & +2-\lambda) \tau \\
& =\left\{-f_{r r}-\left(\frac{s}{c}+\frac{c}{s}\right) f_{r}+\left(\frac{s^{2}}{c^{2}}+\frac{c^{2}}{s^{2}}-4-\lambda\right) f-\frac{1}{s^{2}} f_{\theta \theta}-\frac{1}{c^{2}} f_{\phi \phi}+\frac{2 c}{s^{2}} g_{\theta}+\frac{2 s}{c^{2}} h_{\phi}\right\} \omega_{1} \\
& +\left\{-g_{r r}-\left(\frac{s}{c}+\frac{c}{s}\right) g_{r}+\left(\frac{c^{2}}{s^{2}}-4-\lambda\right) g-\frac{1}{s^{2}} g_{\theta \theta}-\frac{1}{c^{2}} g_{\phi \phi}-\frac{2 c}{s^{2}} f_{\theta}\right\} \omega_{2} \\
& +\left\{-h_{r r}-\left(\frac{s}{c}+\frac{c}{s}\right) h_{r}+\left(\frac{s^{2}}{c^{2}}-4-\lambda\right) h-\frac{1}{s^{2}} h_{\theta \theta}-\frac{1}{c^{2}} h_{\phi \phi}-\frac{2 s}{c^{2}} f_{\phi}\right\} \omega_{3},
\end{aligned}
$$

where $\lambda$ is a real number, the subscripts denote derivatives with respect to the variables $r, \theta$ and $\phi$, and we denote $\sinh r$ and $\cosh r$ by $s$ and $c$, respectively.

We now consider a model space of a neighborhood of a singular locus of a hyperbolic 3-cone-manifold. Let $\alpha, t$ and $l$ be real numbers satisfying that $\alpha>0$ and $l>0$. Let $V$ be a quotient space of $U$ defined by the equivalence relation as follows:

(2) $\left(r_{1}, \theta_{1}, \phi_{1}\right) \sim\left(r_{2}, \theta_{2}, \phi_{2}\right)$

$$
\Longleftrightarrow r_{1}=r_{2} \text { and }{ }^{\exists} k_{1}, k_{2} \in \mathbf{Z} \text { such that } \theta_{1}-\theta_{2}=k_{1} \alpha+k_{2} t, \phi_{1}-\phi_{2}=-k_{2} l \text {. }
$$


Then $V$ is homeomorphic to $(0, \infty) \times T^{2}$, where $T^{2}$ is a 2-dimensional torus. The Riemannian metric $g$ on $U$ induces a Riemannian metric on $V$ which has the same form as $g$. Let $V(R)$ be a subset of $V$ whose elements satisfy the condition that $r=R$. Then $V(R)$ is homeomorphic to $T^{2}$.

Remark. Let $C$ be a hyperbolic 3-cone-manifold with cone-type singularity along a simple closed geodesic $\Sigma$ whose cone angle is equal to $\alpha$ and complex length along $\Sigma$ is equal to $l+t \sqrt{-1}$. Then, if $R$ is sufficiently small, a subset $\bigcup_{0<r<R} V(r)$ of $V$ is a neighborhood of $\Sigma$ in $C-\Sigma$. The Riemannian metric on $C-\Sigma$ is incomplete near $\Sigma$. The metric completion of $C-\Sigma$ is identical to the hyperbolic cone structure on $C$. (See Refs. [1], [6].)

Now, let us assume that $\tau$ can be induced to be a 1-form on $V$. Then it has the following equivariance properties:

$$
\tau(r, \theta+\alpha, \phi)=\tau(r, \theta, \phi) \quad \text { and } \quad \tau(r, \theta, \phi+l)=\tau(r, \theta+t, \phi) .
$$

In order to find solutions of the equation $(\Delta+2-\lambda) \tau=0$, we use separation of variables. We decompose the function $f(r, \theta, \phi)$ into the product of a function $f(r)$ on $(0, \infty)$ and a function on the torus. We decompose $g(r, \theta, \phi)$ and $h(r, \theta, \phi)$ similarly. We further decompose these functions on the torus into eigenfunctions of the Laplacian on the torus, which are of the forms $\cos (a \theta+b \phi)$ and $\sin (a \theta+b \phi)$, where

$$
a:=\frac{2 \pi n}{\alpha} \quad \text { and } \quad b:=\frac{(2 \pi m+a t)}{l} \quad(n, m \in \mathbf{Z})
$$

Such a 1-form $\tau$ is called an 'eigenform' of the Laplacian. Then, from the expression (1), we see that such a 1 -form $\tau$ must be of the type

$$
\tau=f(r) \cos (a \theta+b \phi) \omega_{1}+g(r) \sin (a \theta+b \phi) \omega_{2}+h(r) \sin (a \theta+b \phi) \omega_{3},
$$

or the type

$$
\tau=f(r) \sin (a \theta+b \phi) \omega_{1}+g(r) \cos (a \theta+b \phi) \omega_{2}+h(r) \cos (a \theta+b \phi) \omega_{3} .
$$

Then, for an eigenform $\tau$ of the Laplacian, we can verify the following (see (21) 
in Ref. [4]):

(3)

$$
\begin{aligned}
& (\Delta+2-\lambda) \tau=0 \\
& \Leftrightarrow\left\{\begin{array}{l}
(3.1) f^{\prime \prime}(r)+\left(\frac{s}{c}+\frac{c}{s}\right) f^{\prime}(r)+\left(\lambda-\frac{s^{2}}{c^{2}}-\frac{c^{2}}{s^{2}}-\frac{a^{2}}{s^{2}}-\frac{b^{2}}{c^{2}}\right) f(r) \\
-\frac{2 a c}{s^{2}} g(r)-\frac{2 b s}{c^{2}} h(r)=0, \\
(3.2) g^{\prime \prime}(r)+\left(\frac{s}{c}+\frac{c}{s}\right) g^{\prime}(r)+\left(\lambda-\frac{c^{2}}{s^{2}}-\frac{a^{2}}{s^{2}}-\frac{b^{2}}{c^{2}}\right) g(r)-\frac{2 a c}{s^{2}} f(r)=0, \\
(3.3) h^{\prime \prime}(r)+\left(\frac{s}{c}+\frac{c}{s}\right) h^{\prime}(r)+\left(\lambda-\frac{s^{2}}{c^{2}}-\frac{a^{2}}{s^{2}}-\frac{b^{2}}{c^{2}}\right) h(r)-\frac{2 b s}{c^{2}} f(r)=0,
\end{array}\right.
\end{aligned}
$$

where $f^{\prime}(r)=\frac{d f}{d r}(r)$, etc. Next, we define $z:=\left(\frac{\sinh r}{\cosh r}\right)^{2}$. Then we have

(4)

$$
\begin{aligned}
& (\Delta+2-\lambda) \tau=0 \\
& \Leftrightarrow\left\{\begin{aligned}
(4.1) & 4 z^{2} f^{\prime \prime}(z)+4 z f^{\prime}(z)+\left(\frac{\lambda z}{(1-z)^{2}}-\frac{1}{(1-z)^{2}}-\frac{z^{2}}{(1-z)^{2}}-\frac{a^{2}}{1-z}-\frac{b^{2} z}{1-z}\right) \\
& \times f(z)-\frac{2 a}{(1-z)^{3 / 2}} g(z)-\frac{2 b z^{3 / 2}}{(1-z)^{3 / 2}} h(z)=0, \\
(4.2) & 4 z^{2} g^{\prime \prime}(z)+4 z g^{\prime}(z)+\left(\frac{\lambda z}{(1-z)^{2}}-\frac{1}{(1-z)^{2}}-\frac{a^{2}}{1-z}-\frac{b^{2} z}{1-z}\right) g(z) \\
& -\frac{2 a}{(1-z)^{3 / 2}} f(z)=0, \\
(4.3) & 4 z^{2} h^{\prime \prime}(z)+4 z h^{\prime}(z)+\left(\frac{\lambda z}{(1-z)^{2}}-\frac{z^{2}}{(1-z)^{2}}-\frac{a^{2}}{1-z}-\frac{b^{2} z}{1-z}\right) h(z) \\
& -\frac{2 b z^{3 / 2}}{(1-z)^{3 / 2}} f(z)=0,
\end{aligned}\right.
\end{aligned}
$$

where $f^{\prime}(z)=\frac{d f}{d z}(z)$, etc. Note that $r>0$ is bijectively mapped onto $0<z<1$. The relations $z^{1 / 2}=\frac{\sinh r}{\cosh r}$ and $(1-z)^{1 / 2}=\frac{1}{\cosh r}$ will be helpful to derive (4) from (3). The system (4) can be expressed as
(5) $\left\{\begin{array}{l}(5.1) 2 z^{2}(1-z)^{\frac{3}{2}} P_{1}(a, b, \lambda) f(z)-a g(z)-b z^{\frac{3}{2}} h(z)=0, \\ (5.2) a f(z)=2 z^{2}(1-z)^{\frac{3}{2}} P_{2}(a, b, \lambda) g(z), \\ (5.3) b f(z)=2 z^{\frac{1}{2}}(1-z)^{\frac{3}{2}} P_{3}(a, b, \lambda) h(z),\end{array}\right.$ 
where we define

$$
\begin{aligned}
& P_{1}(a, b, \lambda):=\frac{d^{2}}{d z^{2}}+\frac{1}{z} \frac{d}{d z}+\left(\frac{a^{2}+1}{4 z^{2}(z-1)}+\frac{b^{2}-1}{4 z(z-1)}+\frac{\lambda-2}{4 z(z-1)^{2}}\right) \\
& P_{2}(a, b, \lambda):=\frac{d^{2}}{d z^{2}}+\frac{1}{z} \frac{d}{d z}+\left(\frac{a^{2}+1}{4 z^{2}(z-1)}+\frac{b^{2}}{4 z(z-1)}+\frac{\lambda-1}{4 z(z-1)^{2}}\right) \\
& P_{3}(a, b, \lambda):=\frac{d^{2}}{d z^{2}}+\frac{1}{z} \frac{d}{d z}+\left(\frac{a^{2}}{4 z^{2}(z-1)}+\frac{b^{2}-1}{4 z(z-1)}+\frac{\lambda-1}{4 z(z-1)^{2}}\right) .
\end{aligned}
$$

Now, suppose $\tau$ be the dual 1-form of a harmonic vector field on $V$. Then, by the Weitzenböck formula and the fact that the Ricci curvature of $V$ is -2 , $\tau$ satisfies the differential equation $(\Delta+4) \tau=0$ on $V$. Hence, if $\lambda=-2$, then solutions of the system of ordinary differential equations in (3), (4) or (5) give 1-forms that are dual to harmonic vector fields.

In Sections 3, 4 and 5, we solve the system of differential equations in (5) and present fundamental systems of its solutions in the interval $0<z<1$ that will be denoted by the set of 6 triples $\left\{\left(f_{j}(z), g_{j}(z), h_{j}(z)\right) ; j=1, \ldots, 6\right\}$. Moreover, in Sections 5.1 and 5.2, it is shown that $f_{j}(z), g_{j}(z)$ and $h_{j}(z)$ can be expressed explicitly in terms of hypergeometric functions, if the parameter $a$ satisfies the condition that $a$ is not an integer. Let $f_{j}(r), g_{j}(r)$ and $h_{j}(r)$ be the functions of $r(>0)$ obtained by substituting $\left(\frac{\sinh r}{\cosh r}\right)^{2}$ for $z$ and -2 for $\lambda$ in the functions $f_{j}(z), g_{j}(z)$ and $h_{j}(z)$. Then the main result in this paper is described as follows:

Theorem 2.1. $\quad$ Any harmonic vector field $v$ on $V$ whose dual 1-form $\tau$ is an eigenform of the Laplacian of the type discussed above, which is obtained under the condition $a \notin \mathbf{Z}$, is given by the following linear combination (or the same form with sin and cos interchanged):

$v=\sum_{j=1}^{6}\left\{t_{j} f_{j}(r) \cos (a \theta+b \phi) e_{1}+t_{j} g_{j}(r) \sin (a \theta+b \phi) e_{2}+t_{j} h_{j}(r) \sin (a \theta+b \phi) e_{3}\right\}$,

where $t_{j} \in \mathbf{R}$.

Remark. The variable $z$ is originally taken to be $0<z<1$. However, we can regard all functions of the variable $z$ that appear in this paper to be defined on a simply connected domain in $\mathbf{C}-\{0,1\}$ containing the open interval $(0,1)$. For example, let

$$
\Lambda:=\{z \in \mathbf{R} ; z \leq 0 \text { or } z \geq 1\}=(-\infty, 0] \cup[1, \infty) \subset \mathbf{R},
$$


then $\mathbf{C}-\Lambda=(\mathbf{C}-\mathbf{R}) \cup(0,1)$ is one of such domains. In this domain, we choose the branch of the logarithm such that $-\pi<\operatorname{Im}(\log z)<\pi$ and $-\pi<$ $\operatorname{Im}(\log (1-z))<\pi$. The hypergeometric series $F(\alpha, \beta ; \gamma ; z)$ converges on the unit disk $|z|<1$, and we consider its analytic continuation to $\mathbf{C}-\Lambda$. In the following sections, we regard the domain of all functions of the variable $z$ to be $\mathrm{C}-\Lambda$.

\section{§3. Method of Solving the System of Differential Equations}

In this section we transform the system of differential equations given in (5) into single-component differential equations by eliminating unknown functions and find a representation of the solutions to these single-component differential equations in terms of those of Riemann's $P$-equations.

We consider four cases, determined by whether $a$ and $b$ are zero or nonzero, separately, since the structure of the system (5) is different. In Section 3.1, we study the most general case in which both $a$ and $b$ are nonzero. The reduced cases in which $a=0, b=0$ and $a=b=0$ are treated in Sections 3.2, 3.3 and 3.4 , respectively. We remark that the procedure of the elimination depends on the cases. However, we will see that the solutions of (5) of the case in which $a=0$ can be interpreted as a sort of limit of those of (5) with $a \neq 0$. Similarly for solutions of the cases in which $b=0$ and $a=b=0$. We subdivide Section 3.1 into two parts. In the first part, we obtain a single-component differential equation of 6 -th order which the function $h(z)$ must satisfy and give a representation of its solutions. In the second part, we obtain a singlecomponent differential equation that the function $g(z)$ must satisfy and give a representation of its solutions. By considering both of these differential equations, we find that taking the limits $a \rightarrow 0, b \rightarrow 0$ and $a, b \rightarrow 0$ of the solutions to the system of differential equations in (5) with $a \neq 0, b \neq 0$, these reduce to the solutions obtained from (5) with $a=0, b=0$ and $a=b=0$, respectively.

\begin{tabular}{|c|c|c|c|c|}
\hline Section 3.1: & $a \neq 0, b \neq 0$ & $a \rightarrow 0$ & Section 3.2: & $a=0, b \neq 0$ \\
\hline$b \rightarrow 0$ & & & & $b \rightarrow 0$ \\
\hline Section 3.3: & $a \neq 0, b=0$ & & Section 3.4: & $a=0, b=0$ \\
\hline
\end{tabular}

We now briefly describe the method of solving the single-component differential equation obtained in the first part of Section 3.1. This differential equation is a 6 -th order homogeneous linear differential equation for the function 
$h(z)$ that is obtained by eliminating $f(z)$ and $g(z)$ from the system of differential equations in (5). We first factorize the differential operator of this differential equation (see Theorem 3.1.1). The factors in these factorizations are operators of the type appearing in Riemann's $P$-equation. Moreover, we find (see Theorem 3.1.2) a differential operator which gives a linearly isomorphic mapping from the solution space of Riemann's $P$-equation to a subspace of the solution space of the differential equation in question (see Corollary 3.1.3). This operator is also of the type appearing in Riemann's $P$-equation. In general, this sort of operator is called a 'splitting operator'. (See Ref. [2] for general discussion on factorizations of differential operators of Fuchsian type with three singular points and splitting operators. In Ref. [2], we presented an algorithm for factorizations and also an algorithm for finding splitting operators from factorizations.) Then, we can express solutions of the differential equation under study in terms of Riemann's $P$-equations.

\section{$\S 3.1 . \quad$ The case $a \neq 0$ and $b \neq 0$}

\section{(i) A single-component differential equation for $h(z)$}

We can transform the system of differential equations given in (5) into a single-component differential equation of 6 -th order for the function $h(z)$.

First, we eliminate $f(z)$ from (5.1) and (5.3). Since $b \neq 0$, we obtain the following relation between $g(z)$ and $h(z)$ :

$$
a b g(z)=-4 z^{\frac{5}{2}}(z-1)^{3} S(a, b, \lambda) h(z),
$$

where we define

(7) $S(a, b, \lambda):=z^{\frac{-1}{2}}(1-z)^{\frac{-3}{2}} P_{1}(a, b, \lambda) z^{\frac{1}{2}}(1-z)^{\frac{3}{2}} P_{3}(a, b, \lambda)+\frac{b^{2}}{4} z^{-1}(z-1)^{-3}$.

The differential operator $S(a, b, \lambda)$ is of 4 -th order and its explicit form is given in [3]. It is seen that its coefficients are rational functions of $z$ and even functions with respect to $a$. Next, since $a b \neq 0$, by eliminating $f(z)$ and $g(z)$ from (5.2), (5.3) and (6), we obtain the following equation which $h(z)$ must satisfy in the present, $a \neq 0, b \neq 0$ case:

$$
X(a, b, \lambda) h(z)=0
$$

where $X(a, b, \lambda)$ is a differential operator of 6 -th order defined by 


$$
\begin{aligned}
X(a, b, \lambda):= & z^{\frac{-5}{2}}(1-z)^{-3} P_{2}(a, b, \lambda) z^{\frac{5}{2}}(1-z)^{3} S(a, b, \lambda) \\
& +\frac{a^{2}}{4} z^{-4}(z-1)^{-3} P_{3}(a, b, \lambda) .
\end{aligned}
$$

The coefficients of $X(a, b, \lambda)$ are also rational functions of $z$ and are described concretely in [3]. By referring to [3], it can be seen that the differential equation (8) is of Fuchsian type with regular singularities at $z=0, z=1$ and $z=\infty$ and that its characteristic exponents are

$$
\begin{aligned}
& \text { - } \pm \frac{a}{2}, \frac{2 \pm a}{2}, \frac{4 \pm a}{2} \quad(z=0), \\
& \text { - } \frac{1 \pm \sqrt{2-\lambda}}{2}, \frac{3 \pm \sqrt{2-\lambda}}{2}, \frac{2 \pm \sqrt{3-\lambda}}{2} \quad(z=1), \quad(z=\infty) \\
& \text { - } \frac{-1 \pm b \sqrt{-1}}{2}, \frac{1 \pm b \sqrt{-1}}{2}, \frac{3 \pm b \sqrt{-1}}{2} \quad(z)
\end{aligned}
$$

By direct computation, it can be verified that the theorem below holds.

Theorem 3.1.1. For any $a, b, \lambda \in \mathbf{R}$, the differential operator $X(a, b, \lambda)$ can be factorized as

$$
\begin{aligned}
X(a, b, \lambda) & =P_{6}(a, b, \lambda) P_{5}(a, b, \lambda) P_{4}(a, b, \lambda) \\
& =P_{6}(-a, b, \lambda) P_{5}(-a, b, \lambda) P_{4}(a, b, \lambda),
\end{aligned}
$$

where we define

$$
\begin{aligned}
& P_{4}(a, b, \lambda):=\frac{d^{2}}{d z^{2}}+\left(\frac{1}{z}-\frac{1}{z-1}\right) \frac{d}{d z}+\left(\frac{a^{2}}{4 z^{2}(z-1)}+\frac{b^{2}+1}{4 z(z-1)}+\frac{\lambda+1}{4 z(z-1)^{2}}\right) \\
& P_{5}(a, b, \lambda):=\frac{d^{2}}{d z^{2}}+\left(\frac{2}{z}+\frac{4}{z-1}\right) \frac{d}{d z}+\left(\frac{a(a+2)}{4 z^{2}(z-1)}+\frac{b^{2}+25}{4 z(z-1)}+\frac{\lambda+7}{4 z(z-1)^{2}}\right) \\
& P_{6}(a, b, \lambda):=\frac{d^{2}}{d z^{2}}+\left(\frac{6}{z}+\frac{6}{z-1}\right) \frac{d}{d z}+\left(\frac{(a-6)(a+4)}{4 z^{2}(z-1)}+\frac{b^{2}+121}{4 z(z-1)}+\frac{\lambda+23}{4 z(z-1)^{2}}\right) .
\end{aligned}
$$

It can be checked that the following relationship between $P_{4}(a, b, \lambda)$, $P_{5}(a, b, \lambda)$ and $P_{6}(a, b, \lambda)$ holds.

\section{Theorem 3.1.2. Define}

$$
P_{7}(a, b, \lambda):=\frac{d^{2}}{d z^{2}}+\left(\frac{3}{z}+\frac{3}{z-1}\right) \frac{d}{d z}+\left(\frac{a^{2}-4}{4 z^{2}(z-1)}+\frac{b^{2}+25}{4 z(z-1)}+\frac{\lambda+1}{4 z(z-1)^{2}}\right) .
$$


Then, if $\lambda \neq 2$, the following equality holds for any $a, b \in \mathbf{R}$,

$$
P_{4}(a, b, \lambda) \frac{4}{2-\lambda} z^{2}(z-1)^{4} P_{7}(a, b, \lambda)-\frac{4}{2-\lambda} z^{2}(z-1)^{4} P_{6}(a, b, \lambda) P_{5}(a, b, \lambda)=1 .
$$

By Theorems 3.1.1 and 3.1.2, we find that the operator $\frac{4}{2-\lambda} z^{2}(z-1)^{4}$ $P_{7}(a, b, \lambda)$ provides a splitting of the short exact sequence of the $\mathcal{D}$-modules

$$
0 \longrightarrow \mathcal{D} / \mathcal{D} P_{6} P_{5} \longrightarrow \mathcal{D} / \mathcal{D} X \longrightarrow \mathcal{D} / \mathcal{D} P_{4} \longrightarrow 0
$$

where the operators $P_{i}(a, b, \lambda)$ and $X(a, b, \lambda)$ are abbreviated as $P_{i}$ and $X$, respectively. In fact, the following corollary provides the decomposition of the solution space of the differential equation $X w(z)=0$ corresponding to this splitting of the short exact sequence of the $\mathcal{D}$-modules.

Corollary 3.1.3. Assume that $a \neq 0$ and $\lambda \neq 2$. Then, for any $b \in \mathbf{R}$, each solution of the differential equation

$$
X(a, b, \lambda) w(z)=0
$$

can be written as

$$
w(z)=u(z)+\frac{4}{2-\lambda} z^{2}(z-1)^{4} P_{7}(a, b, \lambda)\left(v^{+}(z)+v^{-}(z)\right),
$$

where $u(z), v^{+}(z)$ and $v^{-}(z)$ are solutions of the equations $P_{4}(a, b, \lambda) u(z)=0$, $P_{5}(a, b, \lambda) v^{+}(z)=0$ and $P_{5}(-a, b, \lambda) v^{-}(z)=0$, respectively. Conversely, if $u(z), v^{+}(z)$ and $v^{-}(z)$ are solutions of the equations $P_{4}(a, b, \lambda) u(z)=0$, $P_{5}(a, b, \lambda) v^{+}(z)=0$ and $P_{5}(-a, b, \lambda) v^{-}(z)=0$, then

$$
w(z):=u(z)+\frac{4}{2-\lambda} z^{2}(z-1)^{4} P_{7}(a, b, \lambda)\left(v^{+}(z)+v^{-}(z)\right)
$$

satisfies the equation $X(a, b, \lambda) w(z)=0$.

Proof. We write the operators $P_{i}(a, b, \lambda), P_{i}(-a, b, \lambda)$ and $X(a, b, \lambda)$ as $P_{i}, P_{i}^{-}$and $X$, respectively. We can rephrase Theorem 3.1.1 as $X=P_{6} P_{5} P_{4}=$ $P_{6}^{-} P_{5}^{-} P_{4}$. By Theorem 3.1.2, we have

$$
\begin{aligned}
\frac{4}{2-\lambda} z^{2}(z-1)^{4} & P_{6} P_{5} P_{4} \frac{4}{2-\lambda} z^{2}(z-1)^{4} P_{7} v(z) \\
& =\frac{4}{2-\lambda} z^{2}(z-1)^{4} P_{6} P_{5}\left(\frac{4}{2-\lambda} z^{2}(z-1)^{4} P_{6} P_{5}+1\right) v(z) \\
& =\left(\frac{4}{2-\lambda} z^{2}(z-1)^{4} P_{6} P_{5}+1\right) \frac{4}{2-\lambda} z^{2}(z-1)^{4} P_{6} P_{5} v(z) .
\end{aligned}
$$


Then, if $P_{6} P_{5} v(z)=0$ holds, so too does $P_{6} P_{5} P_{4} \frac{4}{2-\lambda} z^{2}(z-1)^{4} P_{7} v(z)=0$. Thus we can define the mapping

$$
\Phi:\left\{u(z) ; P_{4} u(z)=0\right\} \oplus\left\{v(z) ; P_{6} P_{5} v(z)=0\right\} \rightarrow\{w(z) ; X w(z)=0\}
$$

by

$$
\Phi(u(z), v(z)):=u(z)+\frac{4}{2-\lambda} z^{2}(z-1)^{4} P_{7} v(z) .
$$

Now, assume that $u(z)+\frac{4}{2-\lambda} z^{2}(z-1)^{4} P_{7} v(z)=0$. Then

$$
\begin{aligned}
0 & =-P_{4} u(z)=P_{4} \frac{4}{2-\lambda} z^{2}(z-1)^{4} P_{7} v(z) \\
& =\left(\frac{4}{2-\lambda} z^{2}(z-1)^{4} P_{6} P_{5}+1\right) v(z)=v(z),
\end{aligned}
$$

which implies that $u(z)=0$ also. This shows that $\Phi$ is injective. Therefore $\Phi$ is a linear isomorphism, because the two spaces $\left\{u(z) ; P_{4} u(z)=0\right\} \oplus$ $\left\{v(z) ; P_{6} P_{5} v(z)=0\right\}$ and $\{w(z) ; X w(z)=0\}$ are both dimension 6 .

By uniqueness of decomposition of differential operators in the ring of operators, we have $P_{6} P_{5}=P_{6}^{-} P_{5}^{-}$. Now, define the mapping

$$
\Psi:\left\{v^{+}(z) ; P_{5} v^{+}(z)=0\right\} \oplus\left\{v^{-}(z) ; P_{5}^{-} v^{-}(z)=0\right\} \rightarrow\left\{v(z) ; P_{6} P_{5} v(z)=0\right\}
$$

by

$$
\Psi\left(v^{+}(z), v^{-}(z)\right):=v^{+}(z)+v^{-}(z) .
$$

If $v^{+}(z)+v^{-}(z)=0$, then from the relation $P_{5}^{-}-P_{5}=\frac{a}{z^{2}(1-z)}$, we obtain

$$
0=P_{5} v^{+}(z)=-P_{5} v^{-}(z)=\left(\frac{a}{z^{2}(1-z)}-P_{5}^{-}\right) v^{-}(z)=\frac{a}{z^{2}(1-z)} v^{-}(z) .
$$

Hence, since $a \neq 0$, we find $v^{-}(z)=0$. This implies, by the above assumption, that $v^{+}(z)=0$. We thus find that $\Psi$ is injective. Therefore, because the two spaces $\left\{v^{+}(z) ; P_{5} v^{+}(z)=0\right\} \oplus\left\{v^{-}(z) ; P_{5}^{-} v^{-}(z)=0\right\}$ and $\left\{v(z) ; P_{6} P_{5} v(z)=0\right\}$ are both dimension $4, \Psi$ is a linear isomorphism. Combining this and the result for $\Phi$, we conclude that

$$
\begin{aligned}
\Phi \circ(1 \oplus \Psi) & :\left\{u(z) ; P_{4} u(z)=0\right\} \oplus\left\{v^{+}(z) ; P_{5} v^{+}(z)=0\right\} \oplus\left\{v^{-}(z) ; P_{5}^{-} v^{-}(z)=0\right\} \\
\rightarrow & \{w(z) ; X w(z)=0\}
\end{aligned}
$$

is also a linear isomorphism. 


\section{(ii) A single-component differential equation for $g(z)$}

We can also obtain a single-component differential equation for $g(z)$ by eliminating $f(z)$ and $h(z)$ from the system of differential equations in (5) as follows.

First, since $a \neq 0$, eliminating the function $f(z)$ from (5.1) and (5.2), we obtain the following relation between $g(z)$ and $h(z)$ :

$$
a b h(z)=-4 z^{\frac{5}{2}}(z-1)^{3} T(a, b, \lambda) g(z),
$$

where we define

$$
T(a, b, \lambda):=z^{-2}(1-z)^{\frac{-3}{2}} P_{1}(a, b, \lambda) z^{2}(1-z)^{\frac{3}{2}} P_{2}(a, b, \lambda)+\frac{a^{2}}{4} z^{-4}(z-1)^{-3} .
$$

The differential operator $T(a, b, \lambda)$ is of 4-th order and described explicitly in [3]. We see that its coefficients are rational functions of $z$ and even functions with respect to $b$. Next, since $a b \neq 0$, by eliminating $f(z)$ and $h(z)$ from (5.2), (5.3) and (16), we obtain the following equation which $g(z)$ must satisfy in the present, $a \neq 0, b \neq 0$ case:

$$
Y(a, b, \lambda) g(z)=0
$$

where $Y(a, b, \lambda)$ is a differential operator of 6 -th order defined by

$$
\begin{aligned}
Y(a, b, \lambda):= & z^{\frac{-5}{2}}(z-1)^{-3} P_{3}(a, b, \lambda) z^{\frac{5}{2}}(z-1)^{3} T(a, b, \lambda) \\
& +\frac{b^{2}}{4} z^{-1}(z-1)^{-3} P_{2}(a, b, \lambda) .
\end{aligned}
$$

The explicit form of $Y(a, b, \lambda)$ is given in [3]. By direct computation, we can verify the following theorem.

Theorem 3.1.4. For any $a, b, \lambda \in \mathbf{R}$, the differential operators $X(a, b, \lambda)$ and $Y(a, b, \lambda)$ satisfy the equality

$$
Y(a, b, \lambda)=z^{\frac{-1}{2}} X(a, b, \lambda) z^{\frac{1}{2}}
$$

By referring to [3], we see that the equation (16) is of Fuchsian type with regular singularities at $z=0, z=1$ and $z=\infty$ and that its characteristic exponents are 


$$
\begin{aligned}
& \text { - } \frac{-1 \pm a}{2}, \frac{1 \pm a}{2}, \frac{3 \pm a}{2} \quad(z=0) \\
& \text { - } \frac{1 \pm \sqrt{2-\lambda}}{2}, \frac{3 \pm \sqrt{2-\lambda}}{2}, \frac{2 \pm \sqrt{3-\lambda}}{2} \quad(z=1)
\end{aligned}
$$$$
\text { - } \pm \frac{b \sqrt{-1}}{2}, \frac{2 \pm b \sqrt{-1}}{2}, \frac{4 \pm b \sqrt{-1}}{2} \quad(z=\infty) \text {. }
$$

We have the following two theorems:

Theorem 3.1.5. For any $a, b, \lambda \in \mathbf{R}$, the differential operator $Y(a, b, \lambda)$ can be factorized as

$$
\begin{aligned}
Y(a, b, \lambda) & =P_{10}(a, b, \lambda) P_{9}(a, b, \lambda) P_{8}(a, b, \lambda) \\
& =P_{10}(a,-b, \lambda) P_{9}(a,-b, \lambda) P_{8}(a, b, \lambda),
\end{aligned}
$$

where we define

$$
\begin{aligned}
P_{8}(a, b, \lambda):= & \frac{d^{2}}{d z^{2}}+\left(\frac{2}{z}-\frac{1}{z-1}\right) \frac{d}{d z}+\left(\frac{a^{2}-1}{4 z^{2}(z-1)}+\frac{b^{2}}{4 z(z-1)}+\frac{\lambda+1}{4 z(z-1)^{2}}\right), \\
P_{9}(a, b, \lambda):= & \frac{d^{2}}{d z^{2}}+\left(\frac{4}{z}+\frac{4}{z-1}\right) \frac{d}{d z} \\
& +\left(\frac{a^{2}-9}{4 z^{2}(z-1)}+\frac{(6+b \sqrt{-1})(8-b \sqrt{-1})}{4 z(z-1)}+\frac{\lambda+7}{4 z(z-1)^{2}}\right) \\
P_{10}(a, b, \lambda):= & \frac{d^{2}}{d z^{2}}+\left(\frac{6}{z}+\frac{6}{z-1}\right) \frac{d}{d z} \\
& +\left(\frac{a^{2}-25}{4 z^{2}(z-1)}+\frac{(12+b \sqrt{-1})(10-b \sqrt{-1})}{4 z(z-1)}+\frac{\lambda+23}{4 z(z-1)^{2}}\right) .
\end{aligned}
$$

Theorem 3.1.6. Define

$$
\begin{aligned}
P_{11}(a, b, \lambda):= & \frac{d^{2}}{d z^{2}}+\left(\frac{7}{z}+\frac{8}{z-1}\right) \frac{d}{d z} \\
& +\left(\frac{a^{2}+2 b \sqrt{-1}-35}{4 z^{2}(z-1)}+\frac{b^{2}+196}{4 z(z-1)}+\frac{\lambda+43}{4 z(z-1)^{2}}\right), \\
P_{12}(a, b, \lambda):= & \frac{d^{2}}{d z^{2}}+\left(\frac{3}{z}+\frac{5}{z-1}\right) \frac{d}{d z} \\
& +\left(\frac{a^{2}+2 b \sqrt{-1}-3}{4 z^{2}(z-1)}+\frac{(6+b \sqrt{-1})(8-b \sqrt{-1})}{4 z(z-1)}+\frac{\lambda+13}{4 z(z-1)^{2}}\right) .
\end{aligned}
$$


Assume that $\lambda \neq 2$. Then, for any $a, b \in \mathbf{R}$, we have

$P_{8}(a, b, \lambda) \frac{4}{\lambda-2} z^{3}(z-1)^{4} P_{12}(a, b, \lambda)-\frac{4}{\lambda-2} z^{3}(z-1)^{4} P_{11}(a, b, \lambda) P_{9}(a, b, \lambda)=1$.

By Theorems 3.1.5 and 3.1.6, we obtain the following corollary which is shown in the same way as Corollary 3.1.3 (see [3] for its proof).

Corollary 3.1.7. Assume that $b \neq 0$ and $\lambda \neq 2$. Then, for any $a \in \mathbf{R}$, each solution of the differential equation

$$
Y(a, b, \lambda) r(z)=0
$$

can be written

$$
r(z)=p(z)+\frac{4}{\lambda-2} z^{3}(z-1)^{4}\left(P_{12}(a, b, \lambda) q^{+}(z)+P_{12}(a,-b, \lambda) q^{-}(z)\right),
$$

where $p(z), q^{+}(z)$ and $q^{-}(z)$ are solutions of the equations $P_{8}(a, b, \lambda) p(z)=0$, $P_{9}(a, b, \lambda) q^{+}(z)=0$ and $P_{9}(a,-b, \lambda) q^{-}(z)=0$, respectively. Conversely, if $p(z)$, $q^{+}(z)$ and $q^{-}(z)$ are solutions of the equations $P_{8}(a, b, \lambda) p(z)=0, P_{9}(a, b, \lambda) q^{+}$ $(z)=0$ and $P_{9}(a,-b, \lambda) q^{-}(z)=0$, then

$$
r(z):=p(z)+\frac{4}{\lambda-2} z^{3}(z-1)^{4}\left(P_{12}(a, b, \lambda) q^{+}(z)+P_{12}(a,-b, \lambda) q^{-}(z)\right)
$$

satisfies the equation $Y(a, b, \lambda) r(z)=0$.

\section{§3.2. The case $a=0$ and $b \neq 0$}

As stated at the beginning of Section 3, the results obtained in Section 3.1 reduce in the $a \rightarrow 0$ limit to those in the present case.

In this case, (5.2) becomes the differential equation

$$
P_{2}(0, b, \lambda) g(z)=0 \text {. }
$$

This is Riemann's $P$-equation and can be solved. Since $b \neq 0$, as in Section 3.1 (i), from (5.1) and (5.3), we obtain the following differential equation of 4 -th order:

$$
S(0, b, \lambda) h(z)=0
$$


By referring to [3], we see that the differential equation (28) is of Fuchsian type with regular singularities at $z=0, z=1$ and $z=\infty$ and that its characteristic exponents are

$$
\begin{array}{ccc}
\bullet 0,0,1,2 & (z=0), \\
\text { - } \frac{1 \pm \sqrt{2-\lambda}}{2}, \frac{2 \pm \sqrt{3-\lambda}}{2} & (z=1), \\
\text { - } \frac{-1 \pm b \sqrt{-1}}{2}, \frac{1 \pm b \sqrt{-1}}{2} & (z=\infty) .
\end{array}
$$

By direct computation, we obtain

Theorem 3.2.1. For any $b, \lambda \in \mathbf{R}$, the differential operator $S(0, b, \lambda)$ can be factorized as

$$
S(0, b, \lambda)=P_{5}(0, b, \lambda) P_{4}(0, b, \lambda)
$$

In analogy to Corollary 3.1.3, by Theorems 3.2.1 and 3.1.2, we obtain the following.

Corollary 3.2.2. Assume that $\lambda \neq 2$. Then, for any $b \in \mathbf{R}$, each solution of the differential equation

$$
S(0, b, \lambda) w(z)=0
$$

can be written as

$$
w(z)=u(z)+\frac{4}{2-\lambda} z^{2}(z-1)^{4} P_{7}(0, b, \lambda) v(z),
$$

where $u(z)$ and $v(z)$ are solutions of the equations $P_{4}(0, b, \lambda) u(z)=0$ and $P_{5}(0, b, \lambda) v(z)=0$, respectively. Conversely, if $u(z)$ and $v(z)$ are solutions of the equations $P_{4}(0, b, \lambda) u(z)=0$ and $P_{5}(0, b, \lambda) v(z)=0$, then

$$
w(z):=u(z)+\frac{4}{2-\lambda} z^{2}(z-1)^{4} P_{7}(0, b, \lambda) v(z)
$$

satisfies the equation $S(0, b, \lambda) w(z)=0$. 
§3.3. The case $a \neq 0$ and $b=0$

As stated at the beginning of Section 3, the results obtained in Section 3.1 reduce in the $b \rightarrow 0$ limit to those in the present case.

In this case, (5.3) becomes the single-component differential equation

$$
P_{3}(a, 0, \lambda) h(z)=0 .
$$

Like (27), this is Riemann's $P$-equation and can be solved. Because $a \neq 0$, as in Section 3.1(ii), from (5.1) and (5.2), we obtain the following differential equation of 4 -th order in $g(z)$ :

$$
T(a, 0, \lambda) g(z)=0 .
$$

Referring to [3], it is seen that the differential equation (34) is of Fuchsian type with regular singularities at $z=0, z=1$ and $z=\infty$ and that its characteristic exponents are

$$
\begin{aligned}
& -\frac{-1 \pm a}{2}, \frac{1 \pm a}{2} \quad(z=0) \\
& \text { - } \frac{1 \pm \sqrt{2-\lambda}}{2}, \frac{2 \pm \sqrt{3-\lambda}}{2} \quad(z=1) \\
& \text { - } 0,0,1,2 \quad(z=\infty)
\end{aligned}
$$

By direct calculation, we have

Theorem 3.3.1. For any $a, \lambda \in \mathbf{R}$, the differential operator $T(a, 0, \lambda)$ can be factorized as

$$
T(a, 0, \lambda)=P_{9}(a, 0, \lambda) P_{8}(a, 0, \lambda) .
$$

In analogy to Corollary 3.1.7, by Theorems 3.3.1 and 3.1.6, we obtain the following.

Corollary 3.3.2. $\quad$ Assume that $\lambda \neq 2$. Then, for any $a \in \mathbf{R}$, each solution of the differential equation

$$
T(a, 0, \lambda) r(z)=0
$$

can be written as

$$
r(z)=p(z)+\frac{4}{\lambda-2} z^{3}(z-1)^{4} P_{12}(a, 0, \lambda) q(z),
$$


where $p(z)$ and $q(z)$ are solutions of the equations $P_{8}(a, 0, \lambda) p(z)=0$ and $P_{9}(a, 0, \lambda) q(z)=0$, respectively. Conversely, if $p(z)$ and $q(z)$ are solutions of the equations $P_{8}(a, 0, \lambda) p(z)=0$ and $P_{9}(a, 0, \lambda) q(z)=0$, then

$$
r(z):=p(z)+\frac{4}{\lambda-2} z^{3}(z-1)^{4} P_{12}(a, 0, \lambda) q(z)
$$

satisfies the equation $T(a, 0, \lambda) r(z)=0$.

\section{§3.4. The case $a=0$ and $b=0$}

As stated at the beginning of Section 3, the results obtained in Section 3.1 reduce in the $a, b \rightarrow 0$ limit to those in the present case.

In this case, the three equations in (5) become the three single-component differential equations

$$
\begin{aligned}
& P_{1}(0,0, \lambda) f(z)=0, \\
& P_{2}(0,0, \lambda) g(z)=0, \\
& P_{3}(0,0, \lambda) h(z)=0,
\end{aligned}
$$

respectively.

\section{$\S 4$. Fundamental Systems of Solutions to the System of Differential Equations}

In this section we present fundamental systems of solutions to the system of differential equations in (5) by making use of the solutions of the singlecomponent differential equations obtained in Section 3. As described in Section 3 , the system of differential equations in (5) depends on whether $a$ and/or $b$ is zero. We obtain fundamental systems of solutions separately in the four cases $a \neq 0$ and $b \neq 0, a=0$ and $b \neq 0, a \neq 0$ and $b=0$, and $a=0$ and $b=0$. Then, we construct a particular representation of the fundamental system of solutions for the case $a \neq 0$ and $b \neq 0$ such that the results in this case reduce to those obtained in the other three cases in the limits that the appropriate parameter(s) goes (go) to zero. 


\section{§4.1. The case $a \neq 0, b \neq 0$ and $\lambda \neq 2$}

(i) System of solutions obtained from $X(a, b, \lambda) h(z)=0$

For a solution $h(z)$ given by Corollary 3.1.3, the corresponding functions $f(z)$ and $g(z)$ are obtained from the relations (5.3) and (6), respectively. These relations are expressed in terms of the operators $P_{3}(a, b, \lambda)$ and $S(a, b, \lambda)$, whose orders are 2 and 4 , respectively. We now show that each of these operators can be reduced to an operator of lower order and then use these new operators to derive a simple expression of solutions to the system of differential equations in $(5)$.

By (5.3) and (6), the components of the solution $(f(z), g(z), h(z))$ that corresponds to a solution $b u(z)$ of the equation $P_{4}(a, b, \lambda) u(z)=0$ are given by

$$
\begin{aligned}
& f(z)=\frac{2}{b} z^{\frac{1}{2}}(1-z)^{\frac{3}{2}} P_{3}(a, b, \lambda) b u(z), \\
& g(z)=\frac{-4}{a b} z^{\frac{5}{2}}(z-1)^{3} S(a, b, \lambda) b u(z), \\
& h(z)=b u(z) .
\end{aligned}
$$

Here, we have

Lemma 4.1.1. If we divide the operators $2 z^{\frac{1}{2}}(1-z)^{\frac{3}{2}} P_{3}(a, b, \lambda)$ and $-4 z^{\frac{5}{2}}(z-1)^{3} S(a, b, \lambda)$ by $P_{4}(a, b, \lambda)$ from the right, then, for any $a, b, \lambda \in \mathbf{R}$, we obtain $R_{1}$ and $a^{2} z^{-\frac{1}{2}}$ as remainders, respectively, where we define

$$
R_{1}:=-2 z^{\frac{1}{2}}(1-z)^{\frac{1}{2}}\left(\frac{d}{d z}-\frac{1}{2(z-1)}\right) .
$$

By Lemma 4.1.1, we obtain

$$
f(z)=R_{1} u(z), \quad g(z)=a z^{-\frac{1}{2}} u(z) \quad \text { and } \quad h(z)=b u(z) .
$$

Note that solutions $(f(z), g(z), h(z))$ of this form satisfy the relation $b z^{\frac{1}{2}} g(z)=$ $a h(z)$.

Next, we derive a representation of solutions that correspond to solutions of the equations $P_{5}(a, b, \lambda) v^{+}(z)=0$ and $P_{5}(-a, b, \lambda) v^{-}(z)=0$. First, note that the composed mapping $\Phi \circ \Psi$ defined in the proof of Corollary 3.1.3 is an isomorphism into its image:

$$
\begin{aligned}
\Phi \circ \Psi: & \left\{v^{+}(z) ; P_{5}(a, b, \lambda) v^{+}(z)=0\right\} \oplus\left\{v^{-}(z) ; P_{5}(-a, b, \lambda) v^{-}(z)=0\right\} \\
& \ni v^{+}(z)+v^{-}(z) \\
\mapsto & \frac{4}{2-\lambda} z^{2}(z-1)^{4} P_{7}(a, b, \lambda)\left(v^{+}(z)+v^{-}(z)\right) \in\{w(z) ; X(a, b, \lambda) w(z)=0\} .
\end{aligned}
$$


Then, by (5.3) and (6), the components of the solution $(f(z), g(z), h(z))$ that corresponds to a solution $\frac{(2-\lambda) b}{4} v^{+}(z)$ of the equation $P_{5}(a, b, \lambda) v^{+}(z)=0$ are given by

$$
\begin{aligned}
& f(z)=\frac{2}{b} z^{\frac{1}{2}}(1-z)^{\frac{3}{2}} P_{3}(a, b, \lambda) \frac{4}{2-\lambda} z^{2}(z-1)^{4} P_{7}(a, b, \lambda) \frac{(2-\lambda) b}{4} v^{+}(z), \\
& g(z)=\frac{-4}{a b} z^{\frac{5}{2}}(z-1)^{3} S(a, b, \lambda) \frac{4}{2-\lambda} z^{2}(z-1)^{4} P_{7}(a, b, \lambda) \frac{(2-\lambda) b}{4} v^{+}(z), \\
& h(z)=\frac{4}{2-\lambda} z^{2}(z-1)^{4} P_{7}(a, b, \lambda) \frac{(2-\lambda) b}{4} v^{+}(z) .
\end{aligned}
$$

Here, we have

Lemma 4.1.2. If we divide the operators $2 z^{\frac{1}{2}}(1-z)^{\frac{3}{2}} P_{3}(a, b, \lambda) z^{2}(z-$ $1)^{4} P_{7}(a, b, \lambda),-4 z^{\frac{5}{2}}(z-1)^{3} S(a, b, \lambda) z^{2}(z-1)^{4} P_{7}(a, b, \lambda)$ and $b z^{2}(z-1)^{4} P_{7}(a, b$, $\lambda)$ by $P_{5}(a, b, \lambda)$ from the right, then, for any $a, b, \lambda \in \mathbf{R}$, we obtain $R_{2}(a, b)$, $a R_{3}(a, \lambda)$ and $R_{4}(a, b)$ as remainders, respectively, where we define

$$
\begin{aligned}
& R_{2}(a, b):=-z^{\frac{1}{2}}(1-z)^{\frac{7}{2}}\left(a \frac{d}{d z}+\frac{a(a+2)}{2 z}+\frac{3 a-a^{2}-b^{2}}{2(z-1)}\right), \\
& R_{3}(a, \lambda):=-z^{\frac{1}{2}}(z-1)^{3}\left(a \frac{d}{d z}+\frac{a(a+2)}{2 z}+\frac{3 a+\lambda-2}{2(z-1)}\right), \\
& R_{4}(a, b):=-b z(z-1)^{3}\left(\frac{d}{d z}+\frac{a+2}{2 z}+\frac{3}{2(z-1)}\right) .
\end{aligned}
$$

Then, by Lemma 4.1.2, we obtain

$$
f(z)=R_{2}(a, b) v^{+}(z), \quad g(z)=R_{3}(a, \lambda) v^{+}(z) \quad \text { and } \quad h(z)=R_{4}(a, b) v^{+}(z) .
$$

Note that solutions of this form satisfy the relation

$$
(2-\lambda)\left(b z^{\frac{1}{2}}(1-z)^{\frac{-1}{2}} f(z)-a h(z)\right)=\left(a^{2}+b^{2}\right)\left(b z^{\frac{1}{2}} g(z)-a h(z)\right) .
$$

In the same manner, with noting that $P_{3}(-a, b, \lambda)=P_{3}(a, b, \lambda), S(-a, b, \lambda)$ $=S(a, b, \lambda)$ and $P_{7}(-a, b, \lambda)=P_{7}(a, b, \lambda)$, we show that the components of each solution $(f(z), g(z), h(z))$ that corresponds to a solution $\frac{(2-\lambda) b}{4} v^{-}(z)$ of the equation $P_{5}(-a, b, \lambda) v^{-}(z)=0$ can be represented in terms of the operators $R_{3}, R_{4}$ and $R_{5}$ as follows:

$$
f(z)=R_{2}(-a, b) v^{-}(z), \quad g(z)=-R_{3}(-a, \lambda) v^{-}(z), \quad h(z)=R_{4}(-a, b) v^{-}(z) .
$$


Summarizing the above, we have the proposition below.

Proposition 4.1.3. Let $\left\{u_{1}(z), u_{2}(z)\right\}, \quad\left\{v_{1}^{+}(z), v_{2}^{+}(z)\right\}$ and $\left\{v_{1}^{-}(z)\right.$, $\left.v_{2}^{-}(z)\right\}$ be fundamental systems of solutions of the equations $P_{4}(a, b, \lambda) u(z)=0$, $P_{5}(a, b, \lambda) v^{+}(z)=0$ and $P_{5}(-a, b, \lambda) v^{-}(z)=0$, respectively. For each $i \in$ $\{1,2\}$, define

$$
\begin{aligned}
\left(f_{i}(z), g_{i}(z), h_{i}(z)\right) & :=\left(R_{1} u_{i}(z), a z^{-\frac{1}{2}} u_{i}(z), b u_{i}(z)\right), \\
\left(f_{i+2}(z), g_{i+2}(z), h_{i+2}(z)\right) & :=\left(R_{2}(a, b) v_{i}^{+}(z), R_{3}(a, \lambda) v_{i}^{+}(z), R_{4}(a, b) v_{i}^{+}(z)\right), \\
\left(f_{i+4}(z), g_{i+4}(z), h_{i+4}(z)\right) & \\
: & =\left(R_{2}(-a, b) v_{i}^{-}(z),-R_{3}(-a, \lambda) v_{i}^{-}(z), R_{4}(-a, b) v_{i}^{-}(z)\right) .
\end{aligned}
$$

Then, in the case where $a \neq 0, b \neq 0$ and $\lambda \neq 2$, the set of 6 triples $\left\{\left(f_{j}(z), g_{j}(z)\right.\right.$, $\left.\left.h_{j}(z)\right) ; j=1, \ldots, 6\right\}$ forms a fundamental system of solutions to the system of differential equations in (5) on the domain $\mathbf{C}-\Lambda$.

Proof. The mapping

$$
\begin{aligned}
\Phi \circ(1 \oplus \Psi): & \left\{u(z) ; P_{4} u(z)=0\right\} \oplus\left\{v^{+}(z) ; P_{5} v^{+}(z)=0\right\} \oplus\left\{v^{-}(z) ; P_{5}^{-} v^{-}(z)=0\right\} \\
\rightarrow & \{w(z) ; X w(z)=0\}
\end{aligned}
$$

given in the proof of Corollary 3.1.3 is a linear isomorphism. We have assumed that $\lambda \neq 2$ and $b \neq 0$. Then the functions $h_{j}(z)$ are linearly independent. Therefore the 6 triples $\left(f_{j}(z), g_{j}(z), h_{j}(z)\right)$ are also linearly independent and thus form a basis of the space of the solutions of (5).

(ii) System of solutions obtained from $Y(a, b, \lambda) g(z)=0$

We now obtain another fundamental system of solutions to the system of differential equations in (5) by making use of the solutions to the singlecomponent differential equation (18), expressed in terms of the function $g(z)$, that are given in Corollary 3.1.7.

By (5.2) and (16), the components of the solution $(f(z), g(z), h(z))$ that corresponds to a solution $a p(z)$ of the equation $P_{8}(a, b, \lambda) p(z)=0$ are given by

$$
\begin{aligned}
f(z) & =\frac{2}{a} z^{2}(1-z)^{\frac{3}{2}} P_{2}(a, b, \lambda) \operatorname{ap}(z), \\
g(z) & =a p(z) \\
h(z) & =\frac{-4}{a b} z^{\frac{5}{2}}(z-1)^{3} T(a, b, \lambda) \operatorname{ap}(z) .
\end{aligned}
$$

Here, we have 
Lemma 4.1.4. If we divide the operators $2 z^{2}(1-z)^{\frac{3}{2}} P_{2}(a, b, \lambda)$ and $-4 z^{\frac{5}{2}}(z-1)^{3} T(a, b, \lambda)$ by $P_{8}(a, b, \lambda)$ from the right, then, for any $a, b, \lambda \in \mathbf{R}$, we obtain $R_{5}$ and $b^{2} z^{\frac{1}{2}}$ as remainders, respectively, where we define

$$
R_{5}:=-2 z(1-z)^{\frac{1}{2}}\left(\frac{d}{d z}-\frac{1}{2 z(z-1)}\right) .
$$

By Lemma 4.1.4, we obtain

$$
f(z)=R_{5} p(z), \quad g(z)=a p(z) \quad \text { and } \quad h(z)=b z^{\frac{1}{2}} p(z) .
$$

Note that solutions $(f(z), g(z), h(z))$ of this form satisfy the relation $b z^{\frac{1}{2}} g(z)=$ $a h(z)$.

Next, we derive a representation of solutions that correspond to solutions of the equations $P_{9}(a, b, \lambda) q^{+}(z)=0$ and $P_{9}(a,-b, \lambda) q^{-}(z)=0$. First, we note that, by Corollary 3.1.7, (5.2) and (16), the components of each solution $(f(z), g(z), h(z))$ that corresponds to a solution $\frac{(\lambda-2) a}{4} q^{+}(z)$ of the equation $P_{9}(a, b, \lambda) q^{+}(z)=0$ are given by

$$
\begin{aligned}
& f(z)=\frac{2}{a} z^{2}(1-z)^{\frac{3}{2}} P_{2}(a, b, \lambda) \frac{4}{\lambda-2} z^{3}(z-1)^{4} P_{12}(a, b, \lambda) \frac{(\lambda-2) a}{4} q^{+}(z), \\
& g(z)=\frac{4}{\lambda-2} z^{3}(z-1)^{4} P_{12}(a, b, \lambda) \frac{(\lambda-2) a}{4} q^{+}(z), \\
& h(z)=\frac{-4}{a b} z^{\frac{5}{2}}(z-1)^{3} T(a, b, \lambda) \frac{4}{\lambda-2} z^{3}(z-1)^{4} P_{12}(a, b, \lambda) \frac{(\lambda-2) a}{4} q^{+}(z) .
\end{aligned}
$$

Here, we have

Lemma 4.1.5. If we divide the operators $2 z^{2}(1-z)^{\frac{3}{2}} P_{2}(a, b, \lambda) z^{3}(z-1)^{4}$ $P_{12}(a, b, \lambda), a z^{3}(z-1)^{4} P_{12}(a, b, \lambda)$ and $-4 z^{\frac{5}{2}}(z-1)^{3} T(a, b, \lambda) z^{3}(z-1)^{4}$ $P_{12}(a, b, \lambda)$ by $P_{9}(a, b, \lambda)$ from the right, then, for any $a, b, \lambda \in \mathbf{R}$, we obtain $R_{6}(a, b), R_{7}(a, b)$ and $b R_{8}(b, \lambda)$ as remainders, respectively, where we define

$$
\begin{aligned}
& R_{6}(a, b):=z^{2}(1-z)^{\frac{7}{2}}\left(b \sqrt{-1} \frac{d}{d z}+\frac{a^{2}+3 b \sqrt{-1}}{2 z}+\frac{3 b \sqrt{-1}-a^{2}-b^{2}}{2(z-1)}\right) \\
& R_{7}(a, b):=a z^{2}(z-1)^{3}\left(\frac{d}{d z}+\frac{b \sqrt{-1}+3}{2 z}+\frac{3}{2(z-1)}\right) \\
& R_{8}(b, \lambda):=z^{\frac{5}{2}}(z-1)^{3}\left(b \frac{d}{d z}+\frac{3 b \sqrt{-1}-b^{2}+\lambda-2}{2 \sqrt{-1} z}+\frac{3 b \sqrt{-1}-\lambda+2}{2 \sqrt{-1}(z-1)}\right) .
\end{aligned}
$$


Then, by Lemma 4.1.5, we obtain

$$
f(z)=R_{6}(a, b) q^{+}(z), \quad g(z)=R_{7}(a, b) q^{+}(z) \quad \text { and } \quad h(z)=R_{8}(b, \lambda) q^{+}(z) .
$$

Note that solutions of this form satisfy the relation

$$
(2-\lambda) \sqrt{-1}\left(a z^{\frac{1}{2}}(1-z)^{\frac{-1}{2}} f(z)+b \sqrt{-1} z^{\frac{1}{2}} g(z)\right)=\left(a^{2}+b^{2}\right)\left(b z^{\frac{1}{2}} g(z)-a h(z)\right) .
$$

In the same manner, with noting that $P_{2}(a,-b, \lambda)=P_{2}(a, b, \lambda)$, and $T(a,-b, \lambda)=T(a, b, \lambda)$, it can be shown that the components of each solution $(f(z), g(z), h(z))$ that corresponds to a solution $\frac{(\lambda-2) a}{4} q^{-}(z)$ of the equation $P_{9}(a,-b, \lambda) q^{-}(z)=0$ can be represented in terms of the operators $R_{6}, R_{7}$ and $R_{8}$ as follows:

$$
f(z)=R_{6}(a,-b) q^{-}(z), \quad g(z)=R_{7}(a,-b) q^{-}(z), \quad h(z)=-R_{8}(-b, \lambda) q^{-}(z) .
$$

Summarizing the above, we have the proposition below, analogous to Proposition 4.1.3.

Proposition 4.1.6. Let $\left\{p_{1}(z), p_{2}(z)\right\}, \quad\left\{q_{1}^{+}(z), q_{2}^{+}(z)\right\}$ and $\left\{q_{1}^{-}(z)\right.$, $\left.q_{2}^{-}(z)\right\}$ be fundamental systems of solutions of the equations $P_{8}(a, b, \lambda) p(z)=0$, $P_{9}(a, b, \lambda) q^{+}(z)=0$ and $P_{9}(a,-b, \lambda) q^{-}(z)=0$, respectively. For each $i \in\{1$, $2\}$, define

$$
\begin{aligned}
\left(f_{i}(z), g_{i}(z), h_{i}(z)\right) & :=\left(R_{5} p_{i}(z), a p_{i}(z), b z^{\frac{1}{2}} p_{i}(z)\right) \\
\left(f_{i+2}(z), g_{i+2}(z), h_{i+2}(z)\right) & :=\left(R_{6}(a, b) q_{i}^{+}(z), R_{7}(a, b) q_{i}^{+}(z), R_{8}(b, \lambda) q_{i}^{+}(z)\right) \\
\left(f_{i+4}(z), g_{i+4}(z), h_{i+4}(z)\right) & \\
: & =\left(R_{6}(a,-b) q_{i}^{-}(z), R_{7}(a,-b) q_{i}^{-}(z),-R_{8}(-b, \lambda) q_{i}^{-}(z)\right) .
\end{aligned}
$$

Then, in the case where $a \neq 0, b \neq 0$ and $\lambda \neq 2$, the set of 6 triples $\left\{\left(f_{j}(z), g_{j}(z)\right.\right.$, $\left.\left.h_{j}(z)\right) ; j=1, \ldots, 6\right\}$ forms a fundamental system of solutions to the system of differential equations in (5) on the domain $\mathbf{C}-\Lambda$.

\section{§4.2. The case $a=0, b \neq 0$ and $\lambda \neq 2$}

As discussed in Section 3.2, the system of differential equations in (5) is equivalent to the two single-component differential equations (27) and (28). The equation (27) for $g(z)$ can be solved independently. It is seen that the case of Section 4.1 reduces to this case, when the parameter $a$ goes to 0 . 
By Corollary 3.2.2, (5.3) and Lemma 4.1.1, the functions $f(z)$ and $h(z)$ that correspond to a solution $b u(z)$ of the equation $P_{4}(0, b, \lambda) u(z)=0$ are

$$
\begin{aligned}
& f(z)=\frac{2}{b} z^{\frac{1}{2}}(1-z)^{\frac{3}{2}} P_{3}(0, b, \lambda) b u(z)=R_{1} u(z), \\
& h(z)=b u(z) .
\end{aligned}
$$

The functions $f(z)$ and $h(z)$ that correspond to a solution $\frac{(2-\lambda) b}{4} v(z)$ of the equation $P_{5}(0, b, \lambda) v(z)=0$ are

$$
\begin{aligned}
& f(z)=\frac{2}{b} z^{\frac{1}{2}}(1-z)^{\frac{3}{2}} P_{3}(0, b, \lambda) \frac{4}{2-\lambda} z^{2}(z-1)^{4} P_{7}(0, b, \lambda) \frac{(2-\lambda) b}{4} v(z), \\
& h(z)=\frac{4}{2-\lambda} z^{2}(z-1)^{4} P_{7}(0, b, \lambda) \frac{(2-\lambda) b}{4} v(z),
\end{aligned}
$$

also by Corollary 3.2.2 and (5.3). Then, by Lemma 4.1.2, we obtain

$$
f(z)=R_{2}(0, b) v(z) \quad \text { and } \quad h(z)=R_{4}(0, b) v(z) .
$$

Summarizing the above, we have the following, which should be compared with Proposition 4.1.3.

Proposition 4.2.1. Let $\left\{u_{1}(z), u_{2}(z)\right\}, \quad\left\{v_{1}(z), v_{2}(z)\right\}$ and $\left\{m_{1}(z)\right.$, $\left.m_{2}(z)\right\}$ be fundamental systems of solutions to the equations $P_{4}(0, b, \lambda) u(z)=0$, $P_{5}(0, b, \lambda) v(z)=0$ and $P_{2}(0, b, \lambda) m(z)=0$, respectively. For each $i \in\{1,2\}$, define

$$
\begin{aligned}
\left(f_{i}(z), g_{i}(z), h_{i}(z)\right) & :=\left(R_{1} u_{i}(z), 0, b u_{i}(z)\right) \\
\left(f_{i+2}(z), g_{i+2}(z), h_{i+2}(z)\right) & :=\left(0, m_{i}(z), 0\right), \\
\left(f_{i+4}(z), g_{i+4}(z), h_{i+4}(z)\right) & :=\left(R_{2}(0, b) v_{i}(z), 0, R_{4}(0, b) v_{i}(z)\right) .
\end{aligned}
$$

Then, in the case where $a=0, b \neq 0$ and $\lambda \neq 2$, the set of 6 triples $\left\{\left(f_{j}(z), g_{j}(z)\right.\right.$, $\left.\left.h_{j}(z)\right) ; j=1, \ldots, 6\right\}$ forms a fundamental system of solutions to the system of differential equations in (5) on the domain $\mathbf{C}-\Lambda$.

Note that the operator $R_{3}(a, \lambda) P_{5}(a, b, \lambda) R_{3}(a, \lambda)^{-1}$ reduces to the differential operator $P_{2}(0, b, \lambda)$ appearing in $(27)$ as $a \rightarrow 0$. This demonstrates the compatibility of the solution $m_{i}$ in Proposition 4.2.1 with the corresponding solution in Proposition 4.1.3. 


\section{§4.3. The case $a \neq 0, b=0$ and $\lambda \neq 2$}

As discussed in Section 3.3, the system of differential equations in (5) is equivalent to the two single-component differential equations (33) and (34). The equation (33) for $h(z)$ can be solved independently. It is seen that the case of Section 4.1 reduces to this case, when the parameter $b$ goes to 0 .

By Corollary 3.3.2, (5.2) and Lemma 4.1.4, the functions $f(z)$ and $g(z)$ that correspond to a solution $a p(z)$ of the equation $P_{8}(a, 0, \lambda) p(z)=0$ are

$$
\begin{aligned}
& f(z)=\frac{2}{a} z^{2}(1-z)^{\frac{3}{2}} P_{2}(a, 0, \lambda) \operatorname{ap}(z)=R_{5} p(z), \\
& g(z)=a p(z) .
\end{aligned}
$$

The functions $f(z)$ and $g(z)$ that correspond to a solution $\frac{(\lambda-2) a}{4} q(z)$ of the equation $P_{9}(a, 0, \lambda) q(z)=0$ are

$$
\begin{aligned}
& f(z)=\frac{2}{a} z^{2}(1-z)^{\frac{3}{2}} P_{2}(a, 0, \lambda) \frac{4}{\lambda-2} z^{3}(z-1)^{4} P_{12}(a, 0, \lambda) \frac{(\lambda-2) a}{4} q(z), \\
& g(z)=\frac{4}{\lambda-2} z^{3}(z-1)^{4} P_{12}(a, 0, \lambda) \frac{(\lambda-2) a}{4} q(z),
\end{aligned}
$$

also by Corollary 3.3.2 and (5.2). Then, by Lemma 4.1.5, we obtain

$$
f(z)=R_{6}(a, 0) q(z) \quad \text { and } \quad g(z)=R_{7}(a, 0) q(z) .
$$

Summarizing the above, we have the following, which should be compared with Proposition 4.1.6.

Proposition 4.3.1. Let $\left\{p_{1}(z), p_{2}(z)\right\},\left\{q_{1}(z), q_{2}(z)\right\}$ and $\left\{n_{1}(z), n_{2}(z)\right\}$ be fundamental systems of solutions to the equations $P_{8}(a, 0, \lambda) p(z)=0, P_{9}(a, 0$, $\lambda) q(z)=0$ and $P_{3}(a, 0, \lambda) n(z)=0$, respectively. For each $i \in\{1,2\}$, define

$$
\begin{aligned}
\left(f_{i}(z), g_{i}(z), h_{i}(z)\right) & :=\left(R_{5} p_{i}(z), a p_{i}(z), 0\right) \\
\left(f_{i+2}(z), g_{i+2}(z), h_{i+2}(z)\right) & :=\left(R_{6}(a, 0) q_{i}(z), R_{7}(a, 0) q_{i}(z), 0\right), \\
\left(f_{i+4}(z), g_{i+4}(z), h_{i+4}(z)\right) & :=\left(0,0, n_{i}(z)\right) .
\end{aligned}
$$

Then, in the case where $a \neq 0, b=0$ and $\lambda \neq 2$, the set of 6 triples $\left\{\left(f_{j}(z), g_{j}(z)\right.\right.$, $\left.\left.h_{j}(z)\right) ; j=1, \ldots, 6\right\}$ forms a fundamental system of solutions to the system of differential equations in (5) on the domain $\mathbf{C}-\Lambda$.

Note that $-R_{8}(-b, \lambda) P_{9}(a,-b, \lambda)\left(-R_{8}(-b, \lambda)\right)^{-1}$ reduces to the differential operator $P_{3}(a, 0, \lambda)$ appearing in $(33)$ as $b \rightarrow 0$. This demonstrates the 
compatibility of the solution $n_{i}$ in Proposition 4.3.1 with the corresponding solution in Proposition 4.1.6.

\section{§4.4. The case $a=0, b=0$}

As discussed in Section 3.4, the system of differential equations in (5) is equivalent to the three single-component differential equations (39), (40) and (41). It is seen that the case of Section 4.1 reduces to this case when both $a$ and $b$ go to 0 . Independence of (39), (40) and (41) leads to the following.

Proposition 4.4.1. Let $\left\{l_{1}(z), l_{2}(z)\right\}, \quad\left\{m_{1}(z), m_{2}(z)\right\}$ and $\left\{n_{1}(z)\right.$, $\left.n_{2}(z)\right\}$ be fundamental systems of solutions to the equations $P_{1}(0,0, \lambda) l(z)=0$, $P_{2}(0,0, \lambda) m(z)=0$ and $P_{3}(0,0, \lambda) n(z)=0$, respectively. For each $i \in\{1,2\}$, define

$$
\begin{aligned}
\left(f_{i}(z), g_{i}(z), h_{i}(z)\right) & :=\left(l_{i}(z), 0,0\right), \\
\left(f_{i+2}(z), g_{i+2}(z), h_{i+2}(z)\right) & :=\left(0, m_{i}(z), 0\right), \\
\left(f_{i+4}(z), g_{i+4}(z), h_{i+4}(z)\right) & :=\left(0,0, n_{i}(z)\right) .
\end{aligned}
$$

Then, in the case where $a=0$ and $b=0$, the set of 6 triples $\left\{\left(f_{j}(z), g_{j}(z), h_{j}(z)\right)\right.$; $j=1, \ldots, 6\}$ forms a fundamental system of solutions to the system of differential equations in (5) on the domain $\mathbf{C}-\Lambda$.

\section{$\S 5 . \quad$ Explicit Expressions of the Fundamental Systems of Solutions}

In this section we derive explicit expressions of the fundamental systems of solutions to the system of differential equations in (5), which are given implicitly in the propositions in Section 4. These expressions are obtained in terms of hypergeometric functions by stipulating the parameter $a$ to be "generic". We say that $a$ is generic if it is not an integer (see Assumptions 5.1.1 and 5.3.1).

§5.1. The case $a \neq 0, b \neq 0$ and $\lambda \neq 2$

We now obtain an explicit expression of the fundamental system $\left\{\left(f_{j}(z)\right.\right.$, $\left.\left.g_{j}(z), h_{j}(z)\right) ; j=1, \ldots, 6\right\}$ of solutions to the system of differential equations in (5) presented implicitly in Proposition 4.1.3. 
In the present case, the characteristic exponents of the equations $P_{4}(a, b$, ג) $u(z)=0, P_{5}(a, b, \lambda) v^{+}(z)=0$ and $P_{5}(-a, b, \lambda) v^{-}(z)=0$ are

- $\frac{a}{2}, \frac{-a}{2}(z=0) ; \frac{2+\sqrt{3-\lambda}}{2}, \frac{2-\sqrt{3-\lambda}}{2}(z=1) ; \frac{-1+b \sqrt{-1}}{2}, \frac{-1-b \sqrt{-1}}{2}(z=\infty)$,

- $\frac{a}{2}, \frac{-a-2}{2}(z=0) ; \frac{-3+\sqrt{2-\lambda}}{2}, \frac{-3-\sqrt{2-\lambda}}{2}(z=1) ; \frac{5+b \sqrt{-1}}{2}, \frac{5-b \sqrt{-1}}{2}(z=\infty)$,

- $\frac{a-2}{2}, \frac{-a}{2}(z=0) ; \frac{-3+\sqrt{2-\lambda}}{2}, \frac{-3-\sqrt{2-\lambda}}{2}(z=1) ; \frac{5+b \sqrt{-1}}{2}, \frac{5-b \sqrt{-1}}{2}(z=\infty)$, respectively. The differences between the two exponents at $z=0$ are $a, a+1$ and $a-1$ in the three cases.

Assumption 5.1.1. The parameter $a$ is not an integer.

This assumption is equivalent to the assumption that none of the values $a$, $-a, a+1,-a-1, a-1$ and $-a+1$ is a negative integer. Under this condition, we can choose fundamental systems of solutions to the three equations above in $\{z \in \mathbf{C}-\Lambda ;|z|<1\}$ explicitly. First, define

$$
\begin{aligned}
& u(z, a, b, \lambda):=z^{\frac{a}{2}}(1-z)^{\frac{2+\sqrt{3-\lambda}}{2}} F\left(\frac{a+1+b \sqrt{-1}+\sqrt{3-\lambda}}{2}, \frac{a+1-b \sqrt{-1}+\sqrt{3-\lambda}}{2} ; a+1 ; z\right), \\
& v(z, a, b, \lambda):=z^{\frac{a}{2}}(1-z)^{\frac{-3+\sqrt{2-\lambda}}{2}} F\left(\frac{a+2+b \sqrt{-1}+\sqrt{2-\lambda}}{2}, \frac{a+2-b \sqrt{-1}+\sqrt{2-\lambda}}{2} ; a+2 ; z\right),
\end{aligned}
$$

where $F(\alpha, \beta ; \gamma ; z)$ is the hypergeometric function. Then we choose the fundamental systems as follows:

$$
\begin{aligned}
u_{1}(z) & :=u(z, a, b, \lambda) \\
& =z^{\frac{a}{2}}(1-z)^{\frac{2+\sqrt{3-\lambda}}{2}} F\left(\frac{a+1+b \sqrt{-1}+\sqrt{3-\lambda}}{2}, \frac{a+1-b \sqrt{-1}+\sqrt{3-\lambda}}{2} ; a+1 ; z\right), \\
u_{2}(z) & :=u(z,-a, b, \lambda) \\
& =z^{\frac{-a}{2}}(1-z)^{\frac{2+\sqrt{3-\lambda}}{2}} F\left(\frac{-a+1+b \sqrt{-1}+\sqrt{3-\lambda}}{2}, \frac{-a+1-b \sqrt{-1}+\sqrt{3-\lambda}}{2} ;-a+1 ; z\right), \\
v_{1}^{+}(z) & :=v(z, a, b, \lambda) \\
& =z^{\frac{a}{2}}(1-z)^{\frac{-3+\sqrt{2-\lambda}}{2}} F\left(\frac{a+2+b \sqrt{-1}+\sqrt{2-\lambda}}{2}, \frac{a+2-b \sqrt{-1}+\sqrt{2-\lambda}}{2} ; a+2 ; z\right), \\
v_{2}^{+}(z) & :=v(z,-a-2, b, \lambda) \\
& =z^{\frac{-a-2}{2}}(1-z)^{\frac{-3+\sqrt{2-\lambda}}{2}} F\left(\frac{-a+b \sqrt{-1}+\sqrt{2-\lambda}}{2}, \frac{-a-b \sqrt{-1}+\sqrt{2-\lambda}}{2} ;-a ; z\right), \\
v_{1}^{-}(z) & :=v(z,-a, b, \lambda) \\
& =z^{\frac{-a}{2}}(1-z)^{\frac{-3+\sqrt{2-\lambda}}{2}} F\left(\frac{-a+2+b \sqrt{-1}+\sqrt{2-\lambda}}{2}, \frac{-a+2-b \sqrt{-1}+\sqrt{2-\lambda}}{2} ;-a+2 ; z\right), \\
v_{2}^{-}(z) & :=v(z, a-2, b, \lambda) \\
& =z^{\frac{a-2}{2}}(1-z)^{\frac{-3+\sqrt{2-\lambda}}{2}} F\left(\frac{a+b \sqrt{-1}+\sqrt{2-\lambda}}{2}, \frac{a-b \sqrt{-1}+\sqrt{2-\lambda}}{2} ; a ; z\right)
\end{aligned}
$$


Remark. Without Assumption 5.1.1, it may be necessary to use a standard procedure in the theory of hypergeometric functions, in which logarithmic terms are employed in constructing the fundamental systems of solutions (see Ref. [5]).

Then, by Proposition 4.1.3, with use of the formula

$$
\frac{d}{d z} F(\alpha, \beta ; \gamma ; z)=\frac{\alpha \beta}{\gamma} F(\alpha+1, \beta+1 ; \gamma+1 ; z),
$$

and employing Assumption 5.1.1, we obtain the following explicit expression for the fundamental system of solutions in $\{z \in \mathbf{C}-\Lambda ;|z|<1\}$ to the system of differential equations in (5):

$$
\begin{aligned}
& f_{1}(z)=R_{1} u_{1}(z)=R_{1} u(z, a, b, \lambda)=f_{1}(z, a, b, \lambda), \\
& g_{1}(z)=a z^{\frac{-1}{2}} u_{1}(z)=a z^{\frac{-1}{2}} u(z, a, b, \lambda)=g_{1}(z, a, b, \lambda), \\
& h_{1}(z)=b u_{1}(z)=b u(z, a, b, \lambda)=h_{1}(z, a, b, \lambda) \text {, } \\
& f_{2}(z)=R_{1} u_{2}(z)=R_{1} u(z,-a, b, \lambda)=f_{1}(z,-a, b, \lambda), \\
& g_{2}(z)=a z^{\frac{-1}{2}} u_{2}(z)=a z^{\frac{-1}{2}} u(z,-a, b, \lambda)=-g_{1}(z,-a, b, \lambda), \\
& h_{2}(z)=b u_{2}(z)=b u(z,-a, b, \lambda)=h_{1}(z,-a, b, \lambda) \text {, } \\
& f_{3}(z)=R_{2}(a, b) v_{1}^{+}(z)=R_{2}(a, b) v(z, a, b, \lambda)=f_{3}(z, a, b, \lambda), \\
& g_{3}(z)=R_{3}(a, \lambda) v_{1}^{+}(z)=R_{3}(a, \lambda) v(z, a, b, \lambda)=g_{3}(z, a, b, \lambda), \\
& h_{3}(z)=R_{4}(a, b) v_{1}^{+}(z)=R_{4}(a, b) v(z, a, b, \lambda)=h_{3}(z, a, b, \lambda) \text {, } \\
& f_{4}(z)=R_{2}(a, b) v_{2}^{+}(z)=R_{2}(a, b) v(z,-a-2, b, \lambda)=f_{4}(z, a, b, \lambda), \\
& g_{4}(z)=R_{3}(a, \lambda) v_{2}^{+}(z)=R_{3}(a, \lambda) v(z,-a-2, b, \lambda)=g_{4}(z, a, b, \lambda), \\
& h_{4}(z)=R_{4}(a, b) v_{2}^{+}(z)=R_{4}(a, b) v(z,-a-2, b, \lambda)=h_{4}(z, a, b, \lambda), \\
& f_{5}(z)=R_{2}(-a, b) v_{1}^{-}(z)=R_{2}(-a, b) v(z,-a, b, \lambda)=f_{3}(z,-a, b, \lambda), \\
& g_{5}(z)=-R_{3}(-a, \lambda) v_{1}^{-}(z)=-R_{3}(-a, \lambda) v(z,-a, b, \lambda)=-g_{3}(z,-a, b, \lambda), \\
& h_{5}(z)=R_{4}(-a, b) v_{1}^{-}(z)=R_{4}(-a, b) v(z,-a, b, \lambda)=h_{3}(z,-a, b, \lambda), \\
& f_{6}(z)=R_{2}(-a, b) v_{2}^{-}(z)=R_{2}(-a, b) v(z, a-2, b, \lambda)=f_{4}(z,-a, b, \lambda),
\end{aligned}
$$




$$
\begin{aligned}
& g_{6}(z)=-R_{3}(-a, \lambda) v_{2}^{-}(z)=-R_{3}(-a, \lambda) v(z, a-2, b, \lambda)=-g_{4}(z,-a, b, \lambda), \\
& h_{6}(z)=R_{4}(-a, b) v_{2}^{-}(z)=R_{4}(-a, b) v(z, a-2, b, \lambda)=h_{4}(z,-a, b, \lambda),
\end{aligned}
$$

where we define

$$
\begin{aligned}
& f_{1}(z, a, b, \lambda):=z^{\frac{a-1}{2}}(1-z)^{\frac{1+\sqrt{3-\lambda}}{2}}(z+a z+\sqrt{3-\lambda} z-a) \\
& \times F\left(\frac{a+1+b \sqrt{-1}+\sqrt{3-\lambda}}{2}, \frac{a+1-b \sqrt{-1}+\sqrt{3-\lambda}}{2} ; a+1 ; z\right) \\
& -\frac{(a+1+b \sqrt{-1}+\sqrt{3-\lambda})(a+1-b \sqrt{-1}+\sqrt{3-\lambda})}{2(a+1)} z^{\frac{a+1}{2}}(1-z)^{\frac{3+\sqrt{3-\lambda}}{2}} \\
& \times F\left(\frac{a+3+b \sqrt{-1}+\sqrt{3-\lambda}}{2}, \frac{a+3-b \sqrt{-1}+\sqrt{3-\lambda}}{2} ; a+2 ; z\right), \\
& g_{1}(z, a, b, \lambda):=a z^{\frac{a-1}{2}}(1-z)^{\frac{2+\sqrt{3-\lambda}}{2}} \\
& \times F\left(\frac{a+1+b \sqrt{-1}+\sqrt{3-\lambda}}{2}, \frac{a+1-b \sqrt{-1}+\sqrt{3-\lambda}}{2} ; a+1 ; z\right), \\
& h_{1}(z, a, b, \lambda):=b z^{\frac{a}{2}}(1-z)^{\frac{2+\sqrt{3-\lambda}}{2}} \\
& \times F\left(\frac{a+1+b \sqrt{-1}+\sqrt{3-\lambda}}{2}, \frac{a+1-b \sqrt{-1}+\sqrt{3-\lambda}}{2} ; a+1 ; z\right), \\
& f_{3}(z, a, b, \lambda):=-\frac{1}{2} z^{\frac{a-1}{2}}(1-z)^{\frac{2+\sqrt{2-\lambda}}{2}} \\
& \times\left(-2 a z-a^{2} z+b^{2} z-a \sqrt{2-\lambda} z+2 a+2 a^{2}\right) \\
& \times F\left(\frac{a+2+b \sqrt{-1}+\sqrt{2-\lambda}}{2}, \frac{a+2-b \sqrt{-1}+\sqrt{2-\lambda}}{2} ; a+2 ; z\right) \\
& -\frac{a(a+2+b \sqrt{-1}+\sqrt{2-\lambda})(a+2-b \sqrt{-1}+\sqrt{2-\lambda})}{4(a+2)} z^{\frac{a+1}{2}}(1-z)^{\frac{4+\sqrt{2-\lambda}}{2}} \\
& \times F\left(\frac{a+4+b \sqrt{-1}+\sqrt{2-\lambda}}{2}, \frac{a+4-b \sqrt{-1}+\sqrt{2-\lambda}}{2} ; a+3 ; z\right), \\
& g_{3}(z, a, b, \lambda):=\frac{1}{2} z^{\frac{a-1}{2}}(1-z)^{\frac{1+\sqrt{2-\lambda}}{2}}\left(2 z-2 a z-2 a^{2} z-a \sqrt{2-\lambda} z-\lambda z+2 a+2 a^{2}\right) \\
& \times F\left(\frac{a+2+b \sqrt{-1}+\sqrt{2-\lambda}}{2}, \frac{a+2-b \sqrt{-1}+\sqrt{2-\lambda}}{2} ; a+2 ; z\right) \\
& +\frac{a(a+2+b \sqrt{-1}+\sqrt{2-\lambda})(a+2-b \sqrt{-1}+\sqrt{2-\lambda})}{4(a+2)} z^{\frac{a+1}{2}}(1-z)^{\frac{3+\sqrt{2-\lambda}}{2}} \\
& \times F\left(\frac{a+4+b \sqrt{-1}+\sqrt{2-\lambda}}{2}, \frac{a+4-b \sqrt{-1}+\sqrt{2-\lambda}}{2} ; a+3 ; z\right), \\
& h_{3}(z, a, b, \lambda):=\frac{b}{2} z^{\frac{a}{2}}(1-z)^{\frac{1+\sqrt{2-\lambda}}{2}}(-2 z-2 a z-\sqrt{2-\lambda} z+2+2 a) \\
& \times F\left(\frac{a+2+b \sqrt{-1}+\sqrt{2-\lambda}}{2}, \frac{a+2-b \sqrt{-1}+\sqrt{2-\lambda}}{2} ; a+2 ; z\right)
\end{aligned}
$$




$$
\begin{aligned}
& +\frac{b(a+2+b \sqrt{-1}+\sqrt{2-\lambda})(a+2-b \sqrt{-1}+\sqrt{2-\lambda})}{4(a+2)} z^{\frac{a+2}{2}}(1-z)^{\frac{3+\sqrt{2-\lambda}}{2}} \\
& \times F\left(\frac{a+4+b \sqrt{-1}+\sqrt{2-\lambda}}{2}, \frac{a+4-b \sqrt{-1}+\sqrt{2-\lambda}}{2} ; a+3 ; z\right), \\
& \begin{aligned}
f_{4}(z, a, b, \lambda):= & -\frac{\left(a^{2}+b^{2}-a \sqrt{2-\lambda}\right)}{2} z^{\frac{-a-1}{2}}(1-z)^{\frac{2+\sqrt{2-\lambda}}{2}} \\
& \times F\left(\frac{-a+b \sqrt{-1}+\sqrt{2-\lambda}}{2}, \frac{-a-b \sqrt{-1}+\sqrt{2-\lambda}}{2} ;-a ; z\right) \\
& +\frac{(-a+b \sqrt{-1}+\sqrt{2-\lambda})(-a-b \sqrt{-1}+\sqrt{2-\lambda})}{4} z^{\frac{-a-1}{2}}(1-z)^{\frac{4+\sqrt{2-\lambda}}{2}} \\
& \times F\left(\frac{-a+2+b \sqrt{-1}+\sqrt{2-\lambda}}{2}, \frac{-a+2-b \sqrt{-1}+\sqrt{2-\lambda}}{2} ;-a+1 ; z\right),
\end{aligned} \\
& g_{4}(z, a, b, \lambda):=\frac{2-\lambda-a \sqrt{2-\lambda}}{2} z^{\frac{-a-1}{2}}(1-z)^{\frac{1+\sqrt{2-\lambda}}{2}} \\
& \times F\left(\frac{-a+b \sqrt{-1}+\sqrt{2-\lambda}}{2}, \frac{-a-b \sqrt{-1}+\sqrt{2-\lambda}}{2} ;-a ; z\right) \\
& -\frac{(-a+b \sqrt{-1}+\sqrt{2-\lambda})(-a-b \sqrt{-1}+\sqrt{2-\lambda})}{4} z^{\frac{-a-1}{2}}(1-z)^{\frac{3+\sqrt{2-\lambda}}{2}} \\
& \times F\left(\frac{-a+2+b \sqrt{-1}+\sqrt{2-\lambda}}{2}, \frac{-a+2-b \sqrt{-1}+\sqrt{2-\lambda}}{2} ;-a+1 ; z\right), \\
& \begin{aligned}
h_{4}(z, a, b, \lambda):= & -\frac{b \sqrt{2-\lambda}}{2} z^{\frac{-a}{2}}(1-z)^{\frac{1+\sqrt{2-\lambda}}{2}} \\
& \times F\left(\frac{-a+b \sqrt{-1}+\sqrt{2-\lambda}}{2}, \frac{-a-b \sqrt{-1}+\sqrt{2-\lambda}}{2} ;-a ; z\right)
\end{aligned} \\
& -\frac{b(-a+b \sqrt{-1}+\sqrt{2-\lambda})(-a-b \sqrt{-1}+\sqrt{2-\lambda})}{4 a} z^{\frac{-a}{2}}(1-z)^{\frac{3+\sqrt{2-\lambda}}{2}} \\
& \times F\left(\frac{-a+2+b \sqrt{-1}+\sqrt{2-\lambda}}{2}, \frac{-a+2-b \sqrt{-1}+\sqrt{2-\lambda}}{2} ;-a+1 ; z\right) .
\end{aligned}
$$

Recalling that the parameters $a$ and $b$ are real numbers, it is easy to demonstrate that the following proposition.

Proposition 5.1.2. If $\lambda \in \mathbf{R}$ with $\lambda<2$ and $z \in \mathbf{C}-\Lambda$, then, for each $i \in\{1, \ldots, 6\}$ we have

$$
f_{i}(\bar{z})=\overline{f_{i}(z)}, g_{i}(\bar{z})=\overline{g_{i}(z)}, h_{i}(\bar{z})=\overline{h_{i}(z)}
$$

In particular, if $0<z<1$, then for each $i \in\{1, \ldots, 6\}$ we have $f_{i}(z), g_{i}(z)$, $h_{i}(z) \in \mathbf{R}$.

\section{$\S 5.2$. The case $a=0, b \neq 0$ and $\lambda \neq 2$}

In this case, the characteristic exponents of the equations $P_{4}(0, b, \lambda) u(z)=$ $0, P_{5}(0, b, \lambda) v(z)=0$ and $P_{2}(0, a, \lambda) m(z)=0$ are

- $0,0(z=0) ; \frac{2+\sqrt{3-\lambda}}{2}, \frac{2-\sqrt{3-\lambda}}{2}(z=1) ; \frac{-1+b \sqrt{-1}}{2}, \frac{-1-b \sqrt{-1}}{2}(z=\infty)$, 
- $0,-1(z=0) ; \frac{-3+\sqrt{2-\lambda}}{2}, \frac{-3-\sqrt{2-\lambda}}{2}(z=1) ; \frac{5+b \sqrt{-1}}{2}, \frac{5-b \sqrt{-1}}{2}(z=\infty)$,

- $\frac{1}{2}, \frac{-1}{2}(z=0) ; \frac{1+\sqrt{2-\lambda}}{2}, \frac{1-\sqrt{2-\lambda}}{2}(z=1) ; \frac{b \sqrt{-1}}{2}, \frac{-b \sqrt{-1}}{2}(z=\infty)$,

respectively. In each case, the difference between the two exponents at $z=0$ is an integer. Then, although the functions $u_{1}(z), v_{1}(z)$ and $m_{1}(z)$ given in Proposition 4.2.1 are chosen explicitly as follows, the other functions $u_{2}(z)$, $v_{2}(z)$ and $m_{2}(z)$ might contain logarithmic terms:

$$
\begin{aligned}
& u_{1}(z):=u(z, 0, b, \lambda)=(1-z)^{\frac{2+\sqrt{3-\lambda}}{2}} F\left(\frac{1+b \sqrt{-1}+\sqrt{3-\lambda}}{2}, \frac{1-b \sqrt{-1}+\sqrt{3-\lambda}}{2} ; 1 ; z\right), \\
& v_{1}(z):=v(z, 0, b, \lambda)=(1-z)^{\frac{-3+\sqrt{2-\lambda}}{2}} F\left(\frac{2+b \sqrt{-1}+\sqrt{2-\lambda}}{2}, \frac{2-b \sqrt{-1}+\sqrt{2-\lambda}}{2} ; 2 ; z\right), \\
& m_{1}(z):=m(z, b, \lambda)=z^{\frac{1}{2}}(1-z)^{\frac{1+\sqrt{2-\lambda}}{2}} F\left(\frac{2+b \sqrt{-1}+\sqrt{2-\lambda}}{2}, \frac{2-b \sqrt{-1}+\sqrt{2-\lambda}}{2} ; 2 ; z\right),
\end{aligned}
$$

where we define

$$
m(z, b, \lambda):=z^{\frac{1}{2}}(1-z)^{\frac{1+\sqrt{2-\lambda}}{2}} F\left(\frac{2+b \sqrt{-1}+\sqrt{2-\lambda}}{2}, \frac{2-b \sqrt{-1}+\sqrt{2-\lambda}}{2} ; 2 ; z\right) .
$$

§5.3. The case $a \neq 0, b=0$ and $\lambda \neq 2$

Here, the characteristic exponents of the equations $P_{8}(a, 0, \lambda) p(z)=0$, $P_{9}(a, 0, \lambda) q(z)=0$ and $P_{3}(a, 0, \lambda) n(z)=0$ are

- $\quad \frac{a-1}{2}, \frac{-a-1}{2}(z=0) ; \frac{2+\sqrt{3-\lambda}}{2}, \frac{2-\sqrt{3-\lambda}}{2}(z=1) ; 0,0(z=\infty)$,

- $\frac{a-3}{2}, \frac{-a-3}{2}(z=0) ; \frac{-3+\sqrt{2-\lambda}}{2}, \frac{-3-\sqrt{2-\lambda}}{2}(z=1) ; 3,4(z=\infty)$,

- $\frac{a}{2}, \frac{-a}{2}(z=0) ; \frac{1+\sqrt{2-\lambda}}{2}, \frac{1-\sqrt{2-\lambda}}{2}(z=1) ; \frac{1}{2}, \frac{-1}{2}(z=\infty)$,

respectively. In each case, the difference between the two exponents at $z=0$ is $a$.

Assumption 5.3.1. The parameter $a$ is not an integer.

Under this condition, we can choose fundamental systems of solutions in $\{z \in \mathbf{C}-\Lambda ;|z|<1\}$ explicitly. First, define

$$
\begin{aligned}
& p(z, a, \lambda):=z^{\frac{a-1}{2}}(1-z) \frac{2+\sqrt{3-\lambda}}{2} F\left(\frac{a+1+\sqrt{3-\lambda}}{2}, \frac{a+1+\sqrt{3-\lambda}}{2} ; a+1 ; z\right), \\
& q(z, a, \lambda):=z^{\frac{a-3}{2}}(1-z) \frac{-3+\sqrt{2-\lambda}}{2} F\left(\frac{a+2+\sqrt{2-\lambda}}{2}, \frac{a+\sqrt{2-\lambda}}{2} ; a+1 ; z\right), \\
& n(z, a, \lambda):=z^{\frac{a}{2}}(1-z) \frac{1+\sqrt{2-\lambda}}{2} F\left(\frac{a+2+\sqrt{2-\lambda}}{2}, \frac{a+\sqrt{2-\lambda}}{2} ; a+1 ; z\right) .
\end{aligned}
$$


Then, we choose the fundamental systems as follows:

$$
\begin{aligned}
p_{1}(z) & :=p(z, a, \lambda) \\
& =z^{\frac{a-1}{2}}(1-z)^{\frac{2+\sqrt{3-\lambda}}{2}} F\left(\frac{a+1+\sqrt{3-\lambda}}{2}, \frac{a+1+\sqrt{3-\lambda}}{2} ; a+1 ; z\right) \\
& =\frac{1}{a} g_{1}(z, a, 0, \lambda), \\
p_{2}(z) & :=p(z,-a, \lambda) \\
& =z^{\frac{-a-1}{2}}(1-z)^{\frac{2+\sqrt{3-\lambda}}{2}} F\left(\frac{-a+1+\sqrt{3-\lambda}}{2}, \frac{-a+1+\sqrt{3-\lambda}}{2} ;-a+1 ; z\right) \\
& =-\frac{1}{a} g_{1}(z,-a, 0, \lambda), \\
q_{1}(z) & :=q(z, a, \lambda) \\
& =z^{\frac{a-3}{2}}(1-z)^{\frac{-3+\sqrt{2-\lambda}}{2}} F\left(\frac{a+2+\sqrt{2-\lambda}}{2}, \frac{a+\sqrt{2-\lambda}}{2} ; a+1 ; z\right), \\
q_{2}(z) & :=q(z,-a, \lambda) \\
& =z^{\frac{-a-3}{2}}(1-z)^{\frac{-3+\sqrt{2-\lambda}}{2}} F\left(\frac{-a+2+\sqrt{2-\lambda}}{2}, \frac{-a+\sqrt{2-\lambda}}{2} ;-a+1 ; z\right), \\
n_{1}(z) & :=n(z, a, \lambda) \\
& =z^{\frac{a}{2}}(1-z)^{\frac{1+\sqrt{2-\lambda}}{2}} F\left(\frac{a+2+\sqrt{2-\lambda}}{2}, \frac{a+\sqrt{2-\lambda}}{2} ; a+1 ; z\right), \\
n_{2}(z) & :=n(z,-a, \lambda) \\
& =z^{\frac{-a}{2}}(1-z)^{\frac{1+\sqrt{2-\lambda}}{2}} F\left(\frac{-a+2+\sqrt{2-\lambda}}{2}, \frac{-a+\sqrt{2-\lambda}}{2} ;-a+1 ; z\right) .
\end{aligned}
$$

Then, by Proposition 4.3.1, with employing Assumption 5.3.1, we obtain the following explicit expression of the fundamental system of solutions in $\{z \in$ $\mathbf{C}-\Lambda ;|z|<1\}$ to the system of differential equations in (5):

$$
\begin{aligned}
& f_{1}(z)=R_{5} p_{1}(z)=R_{5} p(z, a, \lambda)=f_{1}(z, a, 0, \lambda), \\
& g_{1}(z)=a p_{1}(z)=a p(z, a, \lambda)=g_{1}(z, a, 0, \lambda), \\
& h_{1}(z)=0, \\
& f_{2}(z)=R_{5} p_{2}(z)=R_{5} p(z,-a, \lambda)=f_{1}(z,-a, 0, \lambda), \\
& g_{2}(z)=a p_{2}(z)=a p(z,-a, \lambda)=-g_{1}(z,-a, 0, \lambda), \\
& h_{2}(z)=0, \\
& f_{3}(z)=R_{6}(a, 0) q_{1}(z)=R_{6}(a, 0) q(z, a, \lambda)=\widetilde{f}_{3}(z, a, \lambda), \\
& g_{3}(z)=R_{7}(a, 0) q_{1}(z)=R_{7}(a, 0) q(z, a, \lambda)=\widetilde{g}_{3}(z, a, \lambda), \\
& h_{3}(z)=0,
\end{aligned}
$$




$$
\begin{aligned}
& f_{4}(z)=R_{6}(a, 0) q_{2}(z)=R_{6}(a, 0) q(z,-a, \lambda)=\widetilde{f}_{3}(z,-a, \lambda), \\
& g_{4}(z)=R_{7}(a, 0) q_{2}(z)=R_{7}(a, 0) q(z,-a, \lambda)=-\widetilde{g}_{3}(z,-a, \lambda), \\
& h_{4}(z)=0 \\
& f_{5}(z)=0, \\
& g_{5}(z)=0 \\
& h_{5}(z)=n_{1}(z)=n(z, a, \lambda), \\
& f_{6}(z)=0, \\
& g_{6}(z)=0, \\
& h_{6}(z)=n_{2}(z)=n(z,-a, \lambda),
\end{aligned}
$$

where we define

$$
\begin{aligned}
\widetilde{f}_{3}(z, a, \lambda):= & \frac{a^{2}}{2} z^{\frac{a-1}{2}}(1-z)^{\frac{2+\sqrt{2-\lambda}}{2}} F\left(\frac{a+2+\sqrt{2-\lambda}}{2}, \frac{a+\sqrt{2-\lambda}}{2} ; a+1 ; z\right) \\
\widetilde{g}_{3}(z, a, \lambda):= & \frac{a}{2} z^{\frac{a-1}{2}}(1-z)^{\frac{1+\sqrt{2-\lambda}}{2}}(-a+a z+\sqrt{2-\lambda} z) \\
& \times F\left(\frac{a+2+\sqrt{2-\lambda}}{2}, \frac{a+\sqrt{2-\lambda}}{2} ; a+1 ; z\right) \\
& -\frac{a(a+2+\sqrt{2-\lambda})(a+\sqrt{2-\lambda})}{4(a+1)} z^{\frac{a+1}{2}}(1-z)^{\frac{3+\sqrt{2-\lambda}}{2}} \\
& \times F\left(\frac{a+4+\sqrt{2-\lambda}}{2}, \frac{a+2+\sqrt{2-\lambda}}{2} ; a+2 ; z\right) .
\end{aligned}
$$

Proposition 5.3.2. If $\lambda \in \mathbf{R}$ with $\lambda<2$ and $z \in \mathbf{C}-\Lambda$, then, for each $i \in\{1, \ldots, 6\}$ we have

$$
f_{i}(\bar{z})=\overline{f_{i}(z)}, g_{i}(\bar{z})=\overline{g_{i}(z)}, h_{i}(\bar{z})=\overline{h_{i}(z)} .
$$

In particular, if $0<z<1$, then for each $i \in\{1, \ldots, 6\}$ we have $f_{i}(z), g_{i}(z)$, $h_{i}(z) \in \mathbf{R}$.

\section{§5.4. The case $a=0, b=0$}

Here, the characteristic exponents of the equations $P_{1}(0,0, \lambda) l(z)=0$, $P_{2}(0,0, \lambda) m(z)=0$ and $P_{3}(0,0, \lambda) n(z)=0$ are

- $\frac{1}{2}, \frac{-1}{2}(z=0) ; \frac{1+\sqrt{3-\lambda}}{2}, \frac{1-\sqrt{3-\lambda}}{2}(z=1) ; \frac{1}{2}, \frac{-1}{2}(z=\infty)$, 
- $\frac{1}{2}, \frac{-1}{2}(z=0) ; \frac{1+\sqrt{2-\lambda}}{2}, \frac{1-\sqrt{2-\lambda}}{2}(z=1) ; 0,0(z=\infty)$,

- $0,0(z=0) ; \frac{1+\sqrt{2-\lambda}}{2}, \frac{1-\sqrt{2-\lambda}}{2}(z=1) ; \frac{1}{2}, \frac{-1}{2}(z=\infty)$,

respectively. In each case, the difference between the two exponents at $z=0$ is an integer. Then, we can take explicitly the functions $l_{1}(z), m_{1}(z)$ and $n_{1}(z)$ given in Proposition 4.4.1 as follows:

$$
\begin{aligned}
& l_{1}(z):=l_{1}(z, \lambda):=z^{\frac{1}{2}}(1-z)^{\frac{1+\sqrt{3-\lambda}}{2}} F\left(\frac{3+\sqrt{3-\lambda}}{2}, \frac{1+\sqrt{3-\lambda}}{2} ; 2 ; z\right), \\
& m_{1}(z):=m_{1}(z, \lambda):=m(z, 0, \lambda)=z^{\frac{1}{2}}(1-z)^{\frac{1+\sqrt{2-\lambda}}{2}} F\left(\frac{2+\sqrt{2-\lambda}}{2}, \frac{2+\sqrt{2-\lambda}}{2} ; 2 ; z\right), \\
& n_{1}(z):=n_{1}(z, \lambda):=n(z, 0, \lambda)=(1-z)^{\frac{1+\sqrt{2-\lambda}}{2}} F\left(\frac{2+\sqrt{2-\lambda}}{2}, \frac{\sqrt{2-\lambda}}{2} ; 1 ; z\right) .
\end{aligned}
$$

However the other functions $l_{2}(z), m_{2}(z)$ and $n_{2}(z)$ given in Proposition 4.4.1 might contain logarithmic terms. In particular, if $\lambda=-2$, we have

$$
\begin{aligned}
& f_{1}(z)=l_{1}(z)=l_{1}(z,-2)=z^{\frac{1}{2}}(1-z)^{\frac{1+\sqrt{5}}{2}} F\left(\frac{3+\sqrt{5}}{2}, \frac{1+\sqrt{5}}{2} ; 2 ; z\right), \\
& g_{3}(z)=m_{1}(z)=m_{1}(z,-2)=m(z, 0,-2)=z^{\frac{1}{2}}(1-z)^{\frac{-1}{2}}, \\
& h_{5}(z)=n_{1}(z)=n_{1}(z,-2)=n(z, 0,-2)=(1-z)^{\frac{-1}{2}},
\end{aligned}
$$

and we can obtain the other independent solutions of $P_{2}(0,0, \lambda) m(z)=0$ and $P_{3}(0,0, \lambda) n(z)=0$ explicitly as follows:

$$
\begin{aligned}
& g_{4}(z)=m_{2}(z):=z^{\frac{-1}{2}}(1-z)^{\frac{-1}{2}}(1+z \log z), \\
& h_{6}(z)=n_{2}(z):=(1-z)^{\frac{-1}{2}}(z-\log z) .
\end{aligned}
$$

\section{References}

[1] D. Cooper, C. D. Hodgson and S. P. Kerckhoff, Three-dimensional orbifolds and conemanifolds, Math. Soc. Japan, Tokyo, 2000.

[2] M. Fujii and H. Ochiai, An algorithm for solving linear ordinary differential equations of Fuchsian type with three singular points, Interdiscip. Inform. Sci. 9 (2003), no. 1, 189-200.

[3] _ An expression of harmonic vector fields on hyperbolic 3-cone-manifolds in terms of hypergeometric functions - computational details, Kyoto-Math 2006-18. http://www.math.kyoto-u.ac.jp/preprint/2006/18fujii-ochiai.pdf

[4] C. D. Hodgson and S. P. Kerckhoff, Rigidity of hyperbolic cone-manifolds and hyperbolic Dehn surgery, J. Differential Geom. 48 (1998), no. 1, 1-59.

[5] K. Iwasaki, H. Kimura, S. Shimomura and M. Yoshida, From Gauss to Painlevé, Vieweg, Braunschweig, 1991.

[6] S. Kojima, Hyperbolic 3-manifolds singular along knots, Chaos Solitons Fractals 9 (1998), no. 4-5, 765-777.

[7] S. Rosenberg, The Laplacian on a Riemannian manifold, Cambridge Univ. Press, Cambridge, 1997.

[8] E. T. Whittaker and G. N. Watson, A Course of Modern Analysis, Cambridge, 1969. 